\title{
Corrected asymptotics for a multi-server queue in the Halfin-Whitt regime
}

\author{
A.J.E.M. Janssen · J.S.H. van Leeuwaarden • \\ B. Zwart
}

Received: 8 October 2007 / Revised: 9 May 2008 / Published online: 10 June 2008

(C) The Author(s) 2008

\begin{abstract}
To investigate the quality of heavy-traffic approximations for queues with many servers, we consider the steady-state number of waiting customers in an $\mathrm{M} / \mathrm{D} / \mathrm{s}$ queue as $s \rightarrow \infty$. In the Halfin-Whitt regime, it is well known that this random variable converges to the supremum of a Gaussian random walk. This paper develops methods that yield more accurate results in terms of series expansions and inequalities for the probability of an empty queue, and the mean and variance of the queue length distribution. This quantifies the relationship between the limiting system and the queue with a small or moderate number of servers. The main idea is to view the $\mathrm{M} / \mathrm{D} / s$ queue through the prism of the Gaussian random walk: as for the standard Gaussian random walk, we provide scalable series expansions involving terms that include the Riemann zeta function.
\end{abstract}

Keywords M/D/s queue - Halfin-Whitt scaling · Gaussian random walk - All-time maximum · Riemann zeta function · Lerch's transcendent · Spitzer's identity · Queues in heavy traffic · Lambert W Function · Corrected diffusion approximation

Mathematics Subject Classification (2000) $11 \mathrm{M} 06 \cdot 30 \mathrm{~B} 40 \cdot 60 \mathrm{G} 50 \cdot$ 60G51 · $65 \mathrm{~B} 15$

\footnotetext{
A.J.E.M. Janssen

Digital Signal Processing Group, Philips Research, HTC-36 (WO-02), 5656 AE Eindhoven, The Netherlands

e-mail: a.j.e.m.janssen@philips.com

J.S.H. van Leeuwaarden $(\bowtie)$

Mathematics and Computer Science Department, Eindhoven University of Technology, 5612 AZ

Eindhoven, The Netherlands

e-mail: j.s.h.v.leeuwaarden@tue.nl

B. Zwart

H. Milton Stewart School of Industrial and Systems Engineering, Georgia Institute of Technology, 765 Ferst Drive, 30332 Atlanta, USA

e-mail: bertzwart@gatech.edu
} 


\section{Introduction}

Heavy-traffic analysis is a popular tool to analyze stochastic networks, since the analysis of a complicated network often reduces to the analysis of a much simpler (reflected) diffusion, which may be of lower dimension than the original system. This makes the analysis of complex systems tractable, and from a mathematical point of view, these results are appealing since they can be made rigorous.

A downside of heavy-traffic analysis is that the results are of an asymptotic nature, and only form an approximation for a finite-sized system. In a pioneering paper, Siegmund [26] proposed a corrected diffusion approximation for the waiting time in a single-server queue (actually, Siegmund formulated his result in terms of a random walk). In heavy traffic, the workload distribution is approximated by an exponential distribution. Siegmund gave a precise estimate of the correction term, nowadays a classic result and textbook material, cf. Asmussen [2], p. 369. Siegmund's first order correction has been extended recently by Blanchet and Glynn [5], who give a full series expansion for the tail probability of the GI/GI/1 waiting time distribution in heavy traffic.

The results in $[5,26]$ correspond to the conventional heavy-traffic scaling. The present paper considers corrected diffusion approximations for a heavy-traffic scaling known as the Halfin-Whitt [12] regime. This regime considers queues where the number of servers grows large as the system becomes critically loaded. The number of servers $s$ is chosen according to $s=\lambda+\beta \sqrt{\lambda}$, where $\beta$ is some positive constant. As the scaling parameter $\lambda$ tends to infinity, the traffic intensity tends to one according to $1-\mathcal{O}(1 / \sqrt{\lambda})$. The Halfin-Whitt regime is also known as the QED (Quality and Efficiency Driven) regime, due to the fact that, in the limit, a system can be highly utilized (efficiency) while the waiting times stay relatively small (quality). Also, setting the number of servers as $s=\lambda+\beta \sqrt{\lambda}$ is often called square-root staffing. This terminology is motivated by the emergence of large customer contact centers which need to be staffed with agents, thus calling for accurate and scalable approximations of multi-server queues. We refer to Gans et al. [11] and Mandelbaum [21] for overviews.

The Halfin-Whitt regime was formally introduced in [12] for a system with exponential service times $(\mathrm{G} / \mathrm{M} / \mathrm{s}$ queue), although in [17] the same type of scaling was already applied to the Erlang loss model (M/M/s/s queue). The extension of the results on the $\mathrm{G} / \mathrm{M} / s$ queue to non-exponential service times turned out to be challenging. The past few years have witnessed a substantial effort to rise to this challenge, resulting in several papers on the Halfin-Whitt regime for various types of service time distributions, cf. Puhalskii and Reiman [24], Jelenković et al. [18], Whitt [35], Mandelbaum and Momčilovic [20], and Reed [25].

Although these results offer important qualitative insights and are useful to solve concrete staffing problems, one would like to have a better understanding of the quality of the asymptotic approximations. For instance, how fast does convergence to the heavy-traffic limit take place? It would be helpful to have asymptotic estimates or even inequalities from which we could judge just how close the scaled queueing model is to its heavy-traffic limit. Borst et al. [4] consider optimal staffing of an $\mathrm{M} / \mathrm{M} / \mathrm{s}$ queue in the Halfin-Whitt regime, and show numerically that optimizing the 
system based on the Halfin-Whitt approximation (with $s$ infinite instead of finite) of the cost function is rarely off by more than a single agent from systems with as few as 10 servers. As mentioned in the conclusions of [4], these observations call for a theoretical foundation — a task we take up in the present paper.

\subsection{Goals, results and insights}

We now give a general description of the results obtained in this paper. We consider a multi-server queue with arrival rate $\lambda, s$ servers and deterministic service times (set to 1 ). We let the arrival rate of the system grow large and set $s=\lambda+\beta \sqrt{\lambda}$ for some constant $\beta>0$. Our main performance measure is the probability that the queue is empty. The model at hand has been considered before by Jelenković et al. [18] who showed that the scaled number of waiting customers $\hat{Q}_{\lambda}$ converges to the maximum $M_{\beta}$ of a Gaussian random walk with drift $-\beta$, for which the emptiness probability is known. As $\lambda \rightarrow \infty$, for $\beta<2 \sqrt{\pi}$, there is the result

$$
\mathbb{P}\left(\hat{Q}_{\lambda}=0\right) \rightarrow \mathbb{P}\left(M_{\beta}=0\right)=\sqrt{2} \beta \exp \left\{\frac{\beta}{\sqrt{2 \pi}} \sum_{r=0}^{\infty} \frac{\zeta(1 / 2-r)}{r !(2 r+1)}\left(\frac{-\beta^{2}}{2}\right)^{r}\right\},
$$

with $\zeta$ the Riemann zeta function, see Chang and Peres [6], and Janssen and Van Leeuwaarden $[13,14]$. The limiting result for $\mathbb{P}\left(M_{\beta}=0\right)$ has the appealing property that the time to compute it does not depend on the number of servers, which is the case for standard computational procedures for the M/D/s queue, see e.g. Tijms [32] and Franx [10] and references therein.

The main aim of this paper is to obtain series expansions refining this asymptotic result. These series expansions can be used in two ways. First of all, the series expansions quantify the relationship between the limiting system and the queue with a small or moderate number of servers. In addition, the first term (or first few terms) of these expansions have the correct behavior as the number of servers grows large.

One insight we find particularly interesting is that our approximations are not based on the parameter $\beta$, but on a modification of it, which depends on $s$ and is given by

$$
\alpha(s)=(-2 s(1-\rho+\ln \rho))^{1 / 2},
$$

with $\rho=\lambda / s$. This function converges to $\beta$ as $s \rightarrow \infty$, cf. Lemma 7. Another insight we obtain is that the resulting approximation $\mathbb{P}\left(M_{\alpha(s)}=0\right)$ is, in fact, a lower bound for $\mathbb{P}\left(\hat{Q}_{\lambda}=0\right)$. We also obtain an upper bound, again involving the function $\alpha(s)$.

The model we consider may seem rather specific, but one should realize that obtaining series expansions and bounds of this type is by no means a trivial task. The state of the art for traditional corrected diffusion approximations does not go beyond the random walk, and relies on the availability of the Wiener-Hopf factorization. In the Halfin-Whitt regime, the limiting no-wait probability has been found in two cases only, namely for exponential service times and for deterministic service times. We believe that the latter case is the most challenging one.

We apply the methods developed in this paper to the $\mathrm{M} / \mathrm{M} / s$ queue in [15], in which case the Halfin-Whitt regime results in a non-degenerate limit for the Erlang $\mathrm{C}$ 
formula (probability that a customer has to wait). There we obtain the same important insight: the Halfin-Whitt approximation can be substantially improved when $\beta$ is replaced with $\alpha(s)$; this function is the same for both models.

We finally like to point out that the results in this paper are all formulated for the special case of Poisson arrivals, but the methodology we develop is applicable to more general models (see Sect. 6). An additional motivation for considering deterministic service times is that the number of waiting customers in the queue is related to a discrete bulk-service queue, which has proven its relevance in a variety of applications (see [33], Chap. 5, for an overview).

\subsection{Methodology}

We now turn to a discussion and motivation of the techniques we use and develop in this paper. The ratio of $\mathbb{P}\left(\hat{Q}_{\lambda}=0\right)$ and $\mathbb{P}\left(M_{\beta}=0\right)$ serves as a primary measure of convergence and should tend to one as $\lambda$ grows large. This ratio can be expressed as (using Spitzer's identity, cf. (19))

$$
\frac{\mathbb{P}\left(\hat{Q}_{\lambda}=0\right)}{\mathbb{P}\left(M_{\beta}=0\right)}=\exp \left\{\sum_{l=1}^{\infty} \frac{1}{l}\left(\mathbb{P}\left(\hat{A}_{\lambda l} \leq \beta \sqrt{l}\right)-P(\beta \sqrt{l})\right)\right\},
$$

where $\hat{A}_{\lambda l}=\left(A_{l \lambda}-l \lambda\right) / \sqrt{l \lambda}$ and $A_{l \lambda}$ a Poisson random variable with mean $l \lambda$, and

$$
P(x)=\frac{1}{\sqrt{2 \pi}} \int_{-\infty}^{x} e^{-u^{2} / 2} \mathrm{~d} u
$$

the normal distribution function. To estimate (3) one can use Berry-Esseen bounds, but these do not lead to sharp results (cf. Lemma 1). In order to get more precise estimates one can use classical approximations for sums of i.i.d. random variables like saddlepoint approximations or Edgeworth expansions (see [3, 19]). However, these require each quantity $\mathbb{P}\left(\hat{A}_{\lambda l} \leq \beta \sqrt{l}\right)-P(\beta \sqrt{l})$ to be approximated separately and uniformly in $l$.

To get convenient asymptotic expansions, we follow a different approach: we bring $\mathbb{P}\left(\hat{A}_{\lambda l} \leq \beta \sqrt{l}\right)$ into quasi-Gaussian form, a method that is standard in asymptotic analysis (for an illuminating discussion see De Bruijn [8], pp. 67-71). The resulting asymptotic expansion for e.g. the probability of an empty queue then contains terms of the type

$$
G_{k}(a)=\sum_{l=1}^{\infty} l^{k+1 / 2} \int_{a}^{\infty} e^{-\frac{1}{2} l s x^{2}} z(x) \mathrm{d} x, \quad a, s \in \mathbb{R}^{+}, k \in \mathbb{Z},
$$

where $z(x)$ is some function that does not depend on $l$. This approach seems technical at first sight but we believe it to be elegant and even intuitively appealing, as there is a clear interpretation in terms of a change-of-measure argument, see the end of Sect. 2.

A large part of this paper deals with obtaining the quasi-Gaussian form, analyzing $z(x)$, and reformulating and estimating $G_{k}(a)$ which is done in Sect. 4. A key result is Theorem 3, which gives a representation of $G_{k}(a)$ for a large class of functions $z(x)$; 
the only condition that is imposed on $z(x)$ is that $z:[0, \infty) \mapsto \mathbb{C}$ is a continuous function satisfying $z(x)=\mathcal{O}\left(\exp \left(\varepsilon x^{2}\right)\right)$ for any $\varepsilon>0$ and that $z(x)$ has a Taylor series around zero. To illustrate the generality of our result, we note that the result (1) of Chang and Peres on the Gaussian random walk can be viewed as a special case by taking $z(x) \equiv 1$.

We focus on the case in which $A_{\lambda}$ has a Poisson distribution, which ensures a particularly tractable form of $z(x)$ yielding convenient computational schemes. This form is given in Sect. 2.2 and studied in detail in the appendix. The derivative of $z(x)$ is related to the Lambert $W$ function; our treatment is self-contained, produces some important auxiliary results, and is based on earlier results obtained by Szegö [28]. We include our analysis in a separate appendix, since we believe it is interesting in its own right.

Theorem 3 yields a series expansion which can be truncated at a convenient point to obtain high precision estimates of performance measures. Using classical methods, we can even estimate the optimal truncation point of the series expansion. We illustrate these general ideas by specializing them to the $\mathrm{M} / \mathrm{D} / s$ queue in Sect. 4.3.

\subsection{Organization}

This paper is organized as follows. In Sect. 2 we introduce our model and provide short proofs of results which can also be found in [18]. In particular we establish convergence of the number of waiting customers to the maximum of the Gaussian random walk, and give a rough Berry-Esseen bound. These results form a point of departure for the rest of the paper. We also explain in Sect. 2 how our asymptotic analysis will be carried out. In Sect. 3, for the emptiness probability, and the mean and variance of the queue length distribution, we rewrite the Spitzer-type expressions into quasi-Gaussian expressions. The reformulation and estimation of $G_{k}(a)$ is carried out in Sect. 4. Section 5 focuses on lower and upper bounds which have the correct asymptotic behavior in the Halfin-Whitt regime. We use the quasi-Gaussian expression for the emptiness probability obtained in Sect. 3 to derive these bounds. Conclusions and possible extensions are presented in Sect. 6.

\section{The M/D/s queue and the Halfin-Whitt regime}

We consider the $\mathrm{M} / \mathrm{D} / \mathrm{s}$ queue and keep track of the number of customers waiting in the queue (without those in service) at the end of intervals equal to the constant service time (which we set to one). Customers arrive according to a Poisson process with rate $\lambda$ and are served by at most $s$ servers. Let $Q_{\lambda, n}$ denote the number of customers waiting in the queue at the end of interval $n$. The queueing process is then described by

$$
Q_{\lambda, n+1}=\left(Q_{\lambda, n}+A_{\lambda, n}-s\right)^{+}, \quad n=0,1, \ldots,
$$

where $x^{+}=\max \{0, x\}$, and $A_{\lambda, n}$ denotes the number of customers that arrived at the queue during interval $n$. Obviously, the $A_{\lambda, n}$ are i.i.d. for all $n$, and copies of a Poisson random variable $A_{\lambda}$ with mean $\lambda$. It should be noted that due to the assumption of constant service times, the customers which are being served at the end 
of the considered interval should start within this interval, and for the same reason, the customers whose service is completed during this interval should start before its beginning.

Assume that $\mathbb{E} A_{\lambda, n}=\lambda<s$ and let $Q_{\lambda}$ denote the random variable that follows the stationary queue length distribution, i.e., $Q_{\lambda}$ is the weak limit of $Q_{\lambda, n}$. Let

$$
s=\lambda+\beta \sqrt{\lambda}, \quad \beta>0 .
$$

Let $\left\{S_{n}: n \geq 0\right\}$ be a random walk with $S_{0}=0, S_{n}=X_{1}+\cdots+X_{n}$ and $X, X_{1}, X_{2}, \ldots$ i.i.d. random variables with $\mathbb{E} X<0$, and let $M:=\max \left\{S_{n}: n \geq 0\right\}$ denote the alltime maximum. When $X$ is normally distributed with mean $-\beta<0$ and variance 1 we speak of the Gaussian random walk and denote its all-time maximum by $M_{\beta}$. We often use the following notation which is standard in asymptotic analysis:

$$
f(x) \sim \sum_{n=0}^{\infty} f_{n}(x),
$$

by which we denote that, for every fixed integer $k \geq 1$,

$$
f(x)-\sum_{n=0}^{k-1} f_{n}(x)=f_{k}(x)(1+o(1))
$$

Let $\stackrel{d}{\rightarrow}$ denote convergence in distribution.

\subsection{Basic results}

The following theorem can be proved using a similar approach as in Jelenković et al. [18]. We give a separate proof, because our setting is slightly different and more specific.

Theorem 1 Define $\hat{Q}_{\lambda}=Q_{\lambda} / \sqrt{\lambda}$. As $\lambda \rightarrow \infty$,

(i) $\hat{Q}_{\lambda} \stackrel{d}{\rightarrow} M_{\beta}$;

(ii) $\mathbb{P}\left(\hat{Q}_{\lambda}=0\right) \rightarrow \mathbb{P}\left(M_{\beta}=0\right)$;

(iii) $\mathbb{E}\left[\hat{Q}_{\lambda}^{k}\right] \rightarrow \mathbb{E}\left[M_{\beta}^{k}\right]$ for any $k>0$.

Proof Proof of (i): Note that

$$
\hat{Q}_{\lambda} \stackrel{d}{=}\left(\hat{Q}_{\lambda}+\hat{A}_{\lambda}-\beta\right)^{+}
$$

with $\hat{A}_{\lambda}=\left(A_{\lambda}-\lambda\right) / \sqrt{\lambda}$. Since $\hat{A}_{\lambda}$ converges in distribution to the standard normal random variable as $\lambda \rightarrow \infty$, (i) follows from Theorem X.6.1 in Asmussen [2], if the family $\left(\hat{A}_{\lambda}, \lambda \geq 0\right)$ is uniformly integrable. But this follows simply from the fact that $\mathbb{E}\left[\hat{A}_{\lambda}^{2}\right]=1$ for all $\lambda$.

Proof of (ii): The result $\lim \sup _{\lambda \rightarrow \infty} \mathbb{P}\left(\hat{Q}_{\lambda}=0\right) \leq \mathbb{P}\left(M_{\beta}=0\right)$ follows from (i). To show the lim inf, note that from Spitzer's identity (see (16))

$$
\ln \mathbb{P}\left(\hat{Q}_{\lambda}=0\right)=-\sum_{l=1}^{\infty} \frac{1}{l} \mathbb{P}\left(\hat{A}_{l \lambda}>l \beta\right)
$$


Taking the liminf, applying Fatou's lemma, and using that $\mathbb{P}\left(\hat{A}_{l \lambda}>l \beta\right) \rightarrow P(-\beta \sqrt{l})$ yields

$$
\liminf _{\lambda \rightarrow \infty} \ln \mathbb{P}\left(\hat{Q}_{\lambda}=0\right) \geq-\sum_{l=1}^{\infty} \frac{1}{l} P(-\beta \sqrt{l})=\ln \mathbb{P}\left(M_{\beta}=0\right),
$$

which proves (ii). Statement (iii) follows from (i) if we can prove the additional uniform integrability condition $\sup _{\lambda>N} \mathbb{E}\left[\hat{Q}_{\lambda}^{k}\right]<\infty$ for some constant $N$ and any $k$. To prove this, note that the Cramér-Lundberg-Kingman inequality states that $\mathbb{P}\left(\hat{Q}_{\lambda}>x\right) \leq \mathrm{e}^{-s x}$, for any $s>0$ such that $\mathbb{E}\left[e^{s\left(\hat{A}_{\lambda}-\beta\right)}\right] \leq 1$. After some straightforward computation, this inequality can be rewritten into

$$
\lambda\left(e^{s / \sqrt{\lambda}}-1-\frac{s}{\sqrt{\lambda}}\right)-s \beta \leq 0 .
$$

Since $e^{x}-1-x \leq \frac{1}{2} x^{2} e^{x}$, we see that any $s$ is admissible that satisfies

$$
\frac{s^{2}}{2} e^{s / \sqrt{\lambda}}-s \beta \leq 0 \text {. }
$$

It is easy to see that $s=\beta$ satisfies this inequality if $\lambda \geq N:=(\beta / \ln 2)^{2}$. We conclude that

$$
\mathbb{P}\left(\hat{Q}_{\lambda}>x\right) \leq e^{-\beta x}
$$

for any $x \geq 0$ and any $\lambda>N$. The uniform integrability condition

$$
\sup _{\lambda>N} \mathbb{E}\left[\hat{Q}_{\lambda}^{k}\right]<\infty
$$

now follows directly using for example the formula

$$
\mathbb{E}\left[\hat{Q}_{\lambda}^{k}\right]=\int_{0}^{\infty} k x^{k-1} \mathbb{P}\left(\hat{Q}_{\lambda}>x\right) \mathrm{d} x
$$

As a consequence of Theorem 1 we know that $\mathbb{P}\left(Q_{\lambda}=0\right)$ (which equals $\left.\mathbb{P}\left(\hat{Q}_{\lambda}=0\right)\right)$ tends to $\mathbb{P}\left(M_{\beta}=0\right)$ as $\lambda$ tends to infinity. We are interested in how fast the $\mathrm{M} / \mathrm{D} / \mathrm{s}$ queue in the Halfin-Whitt regime approaches the Gaussian random walk, and so we take the ratio of $\mathbb{P}\left(Q_{\lambda}=0\right)$ and $\mathbb{P}\left(M_{\beta}=0\right)$ as our measure of convergence. From Spitzer's identity for random walks (see Theorem 3.1 in [27]) we have

$$
-\ln \mathbb{P}(M=0)=\sum_{l=1}^{\infty} \frac{1}{l} \mathbb{P}\left(S_{l}>0\right),
$$

which gives for the $\mathrm{M} / \mathrm{D} / s$ queue

$$
-\ln \mathbb{P}\left(Q_{\lambda}=0\right)=\sum_{l=1}^{\infty} \frac{1}{l} \mathbb{P}\left(A_{l \lambda}>l s\right)=\sum_{l=1}^{\infty} \frac{1}{l} \sum_{j=l s+1}^{\infty} e^{-l \lambda} \frac{(l \lambda)^{j}}{j !}
$$


where we choose $\lambda$ such that $s=\lambda+\beta \sqrt{\lambda}$ is integer-valued, i.e., $\lambda=\frac{1}{2}(2 s+$ $\left.\beta^{2}-\left(4 s \beta^{2}+\beta^{4}\right)^{1 / 2}\right)$ with $s=1,2, \ldots$ For the Gaussian random walk we have $\ln \mathbb{P}\left(M_{\beta}=0\right)$ as in (12). The following can be proved using a Berry-Esseen bound.

Lemma 1 For $\omega:=\frac{4}{5} \zeta\left(\frac{3}{2}\right) \approx 2.0899$ there are the bounds

$$
\exp \left\{\frac{-\omega}{\sqrt{\lambda}}\right\} \leq \frac{\mathbb{P}\left(Q_{\lambda}=0\right)}{\mathbb{P}\left(M_{\beta}=0\right)} \leq \exp \left\{\frac{\omega}{\sqrt{\lambda}}\right\}
$$

Proof Along the same lines as Theorem 2 in [18]. From (17) and (12) we get

$$
\begin{aligned}
\frac{\mathbb{P}\left(Q_{\lambda}=0\right)}{\mathbb{P}\left(M_{\beta}=0\right)} & =\exp \left\{\sum_{l=1}^{\infty} \frac{1}{l}\left(P(-\beta \sqrt{l})-\mathbb{P}\left(A_{l \lambda}>l s\right)\right)\right\} \\
& \leq \exp \left\{\sum_{l=1}^{\infty} \frac{1}{l}\left|P(-\beta \sqrt{l})-\mathbb{P}\left(A_{l \lambda}>l s\right)\right|\right\} .
\end{aligned}
$$

Rewriting

$$
\left|P(-\beta \sqrt{l})-\mathbb{P}\left(A_{l \lambda}>l s\right)\right|=\left|\mathbb{P}\left(\hat{A}_{\lambda l} \leq \beta \sqrt{l}\right)-P(\beta \sqrt{l})\right|
$$

with $\hat{A}_{\lambda l}=\left(A_{\lambda l}-\lambda l\right) / \sqrt{\lambda l}$ and using the Berry-Esseen bound for the Poisson case (see Michel [22])

$$
\left|\mathbb{P}\left(\hat{A}_{\lambda l} \leq \beta \sqrt{l}\right)-P(\beta \sqrt{l})\right| \leq \min \left\{\frac{4}{5}, \frac{30.6}{1+\beta^{3} l^{3 / 2}}\right\} \frac{1}{\sqrt{l \lambda}} \leq \frac{4}{5 \sqrt{l \lambda}}
$$

yields, upon substituting (21) into (19), the second inequality in (18). The first inequality in (18) follows in a similar way.

We should stress that the occurrence of $\zeta\left(\frac{3}{2}\right)$ in Lemma 1 is unrelated to the result (1) of Chang and Peres [6].

\subsection{Quasi-Gaussian form: motivation and outline}

The bound in (18) does not reveal much information, except that convergence takes place at rate $\mathcal{O}(1 / \sqrt{\lambda})$. In order to get more precise estimates one can use a saddlepoint approximation or an Edgeworth expansion. However, these are not very convenient, as they require each element $\mathbb{P}\left(\hat{A}_{\lambda l} \leq \beta \sqrt{l}\right)-P(\beta \sqrt{l})$ to be approximated separately due to its dependence on $l$. One example would be the Edgeworth expansion for the Poisson distribution (see [3], (4.18) on p. 96)

$$
\mathbb{P}\left(\hat{A}_{l \lambda} \leq \beta \sqrt{l}\right)=P(\beta \sqrt{l})-\frac{1}{6 \sqrt{2 \pi l \lambda}} e^{-\frac{1}{2} \beta^{2} l}\left(\beta^{2} l-1\right)+\mathcal{O}(1 / \lambda l),
$$


which leads to the approximation

$$
\sum_{l=1}^{\infty} \frac{1}{l}\left(\mathbb{P}\left(\hat{A}_{\lambda l} \leq \beta \sqrt{l}\right)-P(\beta \sqrt{l})\right) \approx-\frac{1}{6 \sqrt{2 \pi \lambda}}\left(\sum_{l=1}^{\infty} \frac{\beta^{2}}{l^{1 / 2}} e^{-\frac{1}{2} \beta^{2} l}-\sum_{l=1}^{\infty} \frac{1}{l^{3 / 2}} e^{-\frac{1}{2} \beta^{2} l}\right) .
$$

It may not come as a surprise that (23) is not a good approximation because we neglect all $\mathcal{O}(1 / \lambda l)$ terms in (22). Although including more terms in the Edgeworth expansion is an option, we choose to get more convenient asymptotic expansions for $\mathbb{P}\left(\hat{A}_{\lambda l} \leq \beta \sqrt{l}\right)$ by bringing it into quasi-Gaussian form.

Specifically, we prove the following theorem in Sect. 3.

\section{Theorem 2}

$$
-\ln \mathbb{P}\left(Q_{\lambda}=0\right)=\sum_{l=1}^{\infty} \frac{p(l s)}{l} \frac{1}{\sqrt{2 \pi}} \int_{\alpha \sqrt{l}}^{\infty} e^{-x^{2} / 2} y^{\prime}(x / \sqrt{l s}) \mathrm{d} x,
$$

in which

$$
\alpha=\left(-2 s\left(1-\frac{\lambda}{s}+\ln \frac{\lambda}{s}\right)\right)^{1 / 2},
$$

$\alpha \rightarrow \beta$ as $\lambda \rightarrow \infty$

$$
p(n)=n^{n} e^{-n} \sqrt{2 \pi n} / n !,
$$

and $y^{\prime}$ is a function analytic in $|x|<2 \sqrt{\pi}$ (see (140)).

For $p$ there is Stirling's formula, see Abramowitz-Stegun [1], 6.1.37 on p. 257,

$$
p(n) \sim 1-\frac{1}{12 n}+\frac{1}{288 n^{2}}+\cdots=\sum_{k=0}^{\infty} \frac{p_{k}}{n^{k}}, \quad n \rightarrow \infty,
$$

and for $y^{\prime}$ there is the power series representation

$$
y^{\prime}(x)=1-\frac{2}{3} x+\frac{1}{12} x^{2}+\cdots=\sum_{i=0}^{\infty} b_{i} x^{i}, \quad|x|<2 \sqrt{\pi} .
$$

From an aesthetic viewpoint, expression (24) conveys much understanding about the character of the convergence, since we have complete agreement with the Gaussian random walk (12) when we would have $\lambda \rightarrow \infty$. The deviations from the quasiGaussian random walk are embodied by $p \not \equiv 1, y^{\prime} \not \equiv 1$ and $\alpha \not \equiv \beta$. From (24) we see that there is the asymptotic expansion

$$
-\ln \mathbb{P}\left(Q_{\lambda}=0\right) \sim \frac{1}{\sqrt{2 \pi}} \sum_{k=0}^{\infty} p_{k} s^{-k+1 / 2} G_{-(k+1)}(\alpha / \sqrt{s}),
$$

where

$$
G_{k}(a)=\sum_{l=1}^{\infty} l^{k+1 / 2} \int_{a}^{\infty} e^{-\frac{1}{2} l s x^{2}} y^{\prime}(x) \mathrm{d} x .
$$


Fig. 1 The function $y^{\prime}(x)$ for $x \in[-2,4]$

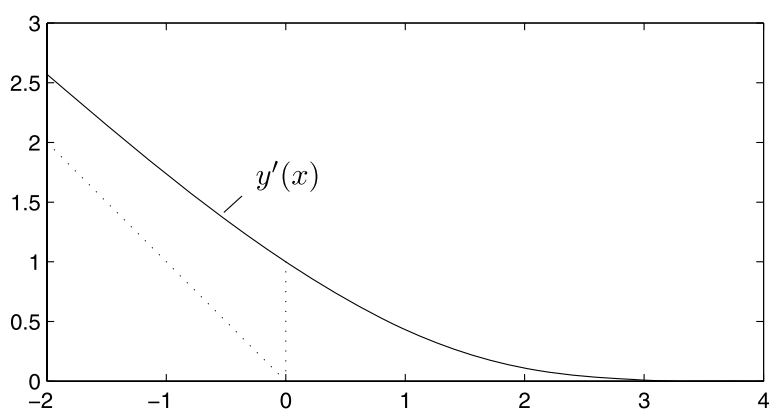

Similar expressions, though somewhat more complicated than the one in (29), exist for $\mathbb{E} Q_{\lambda}$ and $\operatorname{Var} Q_{\lambda}$ (see Sect. 3.2) and these involve $G_{k}$ with $k=0,-1,-2 \ldots$ and $k=1,0,-1 \ldots$, respectively. We shall study $G_{k}$ thoroughly, leading to series expansions, asymptotics and bounds.

We close this section by giving an interpretation of the quasi-Gaussian form (24). Using, see (134),

$$
\frac{1}{p(n)}=\sqrt{\frac{n}{2 \pi}} \int_{-\infty}^{\infty} e^{-\frac{1}{2} n x^{2}} y^{\prime}(x) \mathrm{d} x
$$

and

$$
\int_{\alpha \sqrt{l}}^{\infty} e^{-x^{2} / 2} y^{\prime}(x / \sqrt{l s}) \mathrm{d} x=\sqrt{l s} \int_{\alpha / \sqrt{s}}^{\infty} e^{-l s x^{2} / 2} y^{\prime}(x) \mathrm{d} x
$$

we find from (24) that

$$
-\ln \mathbb{P}\left(Q_{\lambda}=0\right)=\sum_{l=1}^{\infty} \frac{1}{l} \frac{\int_{\alpha}^{\infty} e^{-l x^{2} / 2} y^{\prime}(x / \sqrt{s}) \mathrm{d} x}{\int_{-\infty}^{\infty} e^{-l x^{2} / 2} y^{\prime}(x / \sqrt{s}) \mathrm{d} x}
$$

As mentioned in the introduction, the resulting formula reveals that the summands of the random walk associated with the $\mathrm{M} / \mathrm{D} / \mathrm{s}$ queue, and the summands of the Gaussian random walk, are absolutely continuous with respect to each other. The connecting measure between the two densities has a density as well, and equals $y^{\prime}(\cdot / \sqrt{s})$. Another interpretation is that $\mathbb{P}\left(\hat{Q}_{\lambda}=0\right)$ is obtained by twisting the Gaussian distribution associated with $M_{\beta}$. The associated Radon-Nikodym derivative can again be described in terms of $y^{\prime}(\cdot / \sqrt{s})$.

\section{From Spitzer-type expressions to quasi-Gaussian forms}

In this section we show how to obtain the expression (24). In addition, we present similar results for the mean and variance of the queue length. 


\subsection{Proof of Theorem 2}

For $n=0,1, \ldots$ we let

$$
s_{n}(z)=\sum_{k=0}^{n} \frac{z^{k}}{k !}, \quad z \in \mathbb{C} .
$$

With $\rho=\lambda / s$ and $n=l s$ (so that $\lambda l=n \rho$ ), and

$$
q(\xi)=e^{1-\xi} \xi, \quad \xi \in \mathbb{C},
$$

we then have from Szegö [28], p. 50 (also see Abramowitz-Stegun [1], 6.5.13 on p. 262),

$$
\sum_{j=n+1}^{\infty} e^{-l \lambda} \frac{(l \lambda)^{j}}{j !}=1-e^{-\lambda l} s_{n}(\lambda l)=\frac{n^{n+1} e^{-n}}{n !} \int_{0}^{\rho} q^{n}(\xi) \mathrm{d} \xi .
$$

Using this relation we can rewrite the Spitzer-type expression (17) as

$$
-\ln \mathbb{P}\left(Q_{\lambda}=0\right)=s^{1 / 2} \sum_{l=1}^{\infty} \frac{p(l s)}{\sqrt{2 \pi l}} \int_{0}^{\rho} q^{l s}(\xi) \mathrm{d} \xi
$$

with $p(n)$ as defined in (27). We then consider the equation

$$
f(y):=-\ln q(1-y)=\frac{1}{2} x^{2},
$$

with $x \in \mathbb{C}$ from which $y$ is to be solved. We note that

$$
f(y)=\frac{1}{2} y^{2}+\frac{1}{3} y^{3}+\frac{1}{4} y^{4}+\cdots,
$$

whence there is an analytic solution $y(x)$ around $x=0$ that satisfies $y(x)=$ $x+\mathcal{O}\left(x^{2}\right)$ as $x \rightarrow 0$. Furthermore, since $f$ increases from 0 to $\infty$ as $y$ increases from 0 to 1 , we have that $y(x)$ increases from 0 to $\infty$, and for any $x \geq 0$ there is a unique non-negative solution $y(x)=y$ of (38). Furthermore, we let

$$
\gamma=-2(1-\rho+\ln \rho), \quad \alpha=(s \gamma)^{1 / 2} .
$$

Then it holds that

$$
q^{l s}(\rho)=e^{-\frac{1}{2} l s \gamma}=e^{-\frac{1}{2} \alpha^{2} l}
$$

and

$$
\int_{0}^{\rho} q^{l s}(\xi) \mathrm{d} \xi=\int_{\sqrt{\gamma}}^{\infty} e^{-\frac{1}{2} l s x^{2}} y^{\prime}(x) \mathrm{d} x=\frac{1}{\sqrt{l s}} \int_{\alpha \sqrt{l}}^{\infty} e^{-\frac{1}{2} x^{2}} y^{\prime}(x / \sqrt{l s}) \mathrm{d} x .
$$

Substituting (42) into (37) yields (24). 
Table 1 Interrelations between some parameters and functions

$$
\begin{aligned}
& s=\lambda+\beta \sqrt{\lambda} \\
& \rho=\lambda / s \\
& \gamma=-2(1-\rho+\ln \rho) \\
& \alpha=\sqrt{s \gamma} \\
& a=\alpha / \sqrt{s}=\sqrt{\gamma} \\
& y(x)=x-\frac{1}{3} x^{2}+\frac{1}{36} x^{3}+\cdots ; \quad|x|<2 \sqrt{\pi} \\
& \beta=\sqrt{s} y(\alpha / \sqrt{s})(1-y(\alpha / \sqrt{s}))^{-1 / 2} \\
& \beta=\alpha+\frac{1}{6} s^{-1 / 2} \alpha^{2}+\frac{5}{72} s^{-1} \alpha^{3}+\cdots ; \quad|\alpha / \sqrt{s}|<2 \sqrt{\pi} \\
& p(n)=n^{n} e^{-n} \sqrt{2 \pi n} / n ! \sim 1+\frac{1}{12} n^{-1}+\frac{1}{288} n^{-2}+\cdots
\end{aligned}
$$

Lemma 2 The parameters $\alpha$ and $\beta$ are related according to

$$
\beta / \sqrt{s}=\frac{y(\alpha / \sqrt{s})}{(1-y(\alpha / \sqrt{s}))^{1 / 2}} .
$$

Proof Follows from $1-\rho=y\left(\gamma^{1 / 2}\right)=y(\alpha / \sqrt{s})$, see Table 1 , and

$$
1-\rho=\frac{s-\lambda}{s}=\frac{s-\lambda}{\sqrt{\lambda}}\left(\frac{\lambda}{s}\right)^{1 / 2} \frac{1}{\sqrt{s}}=\frac{\beta}{\sqrt{s}} \rho^{1 / 2}=\frac{\beta}{\sqrt{s}}(1-y(\alpha / \sqrt{s}))^{1 / 2} .
$$

We have that $y(x)(1-y(x))^{-1 / 2}=x+\frac{1}{6} x^{2}+\frac{5}{72} x^{3}+\cdots$ (see the appendix), and hence

$$
\beta=\alpha+\frac{1}{6 \sqrt{s}} \alpha^{2}+\frac{5}{72 s} \alpha^{3}+\cdots
$$

\subsection{Mean and variance of the queue length}

Our primary characteristic in this paper is the probability of an empty queue. However, the techniques that we develop can be applied to other characteristics like the mean and variance of the queue length. From Spitzer's identity it follows that the mean and variance of the maximum $M$ are given by $\sum_{l=1}^{\infty} \frac{1}{l} \mathbb{E}\left(\left(S_{l}^{+}\right)^{k}\right)$ with $k=1$ and $k=2$, respectively. For the $\mathrm{M} / \mathrm{D} / s$ queue this yields

$$
\begin{aligned}
\mathbb{E} Q_{\lambda} & =\sum_{l=1}^{\infty} \frac{1}{l} \sum_{j=l s+1}^{\infty}(j-l s) e^{-l \lambda} \frac{(l \lambda)^{j}}{j !} \\
\operatorname{Var} Q_{\lambda} & =\sum_{l=1}^{\infty} \frac{1}{l} \sum_{j=l s+1}^{\infty}(j-l s)^{2} e^{-l \lambda} \frac{(l \lambda)^{j}}{j !} .
\end{aligned}
$$

This leads after considerable rewriting to

$$
\mathbb{E} Q_{\lambda}=s^{1 / 2} \sum_{l=1}^{\infty} \frac{p(l s)}{\sqrt{2 \pi l}}\left(\rho q^{l s}(\rho)-l s(1-\rho) \int_{0}^{\rho} q^{l s}(\xi) \mathrm{d} \xi\right)
$$


and

$$
\begin{aligned}
\operatorname{Var} Q_{\lambda}= & s^{1 / 2} \sum_{l=1}^{\infty} \frac{p(l s)}{\sqrt{2 \pi l}}\left(-\rho(l s(1-\rho)-1) q^{l s}(\rho)\right. \\
& \left.+\left((1-\rho)^{2} l^{2} s^{2}+l s \rho\right) \int_{0}^{\rho} q^{l s}(\xi) \mathrm{d} \xi\right) .
\end{aligned}
$$

In a similar way as for $\mathbb{P}\left(Q_{\lambda}=0\right)$, (48) and (49) can then be brought into the forms

$$
\begin{aligned}
\mathbb{E} Q_{\lambda}= & \sqrt{s}\left[\sum_{l=1}^{\infty} \rho p(l s) \frac{e^{-\frac{1}{2} \alpha^{2} l}}{\sqrt{2 \pi l}}\right. \\
& \left.-\sum_{l=1}^{\infty} \alpha R(\rho) \frac{p(l s)}{\sqrt{2 \pi}} \int_{\alpha \sqrt{l}}^{\infty} e^{-x^{2} / 2} y^{\prime}(x / \sqrt{l s}) \mathrm{d} x\right] \\
\operatorname{Var} Q_{\lambda}= & s\left[\sum_{l=1}^{\infty}\left(\alpha^{2} l R^{2}(\rho)+\rho\right) \frac{p(l s)}{\sqrt{2 \pi}} \int_{\alpha \sqrt{l}}^{\infty} e^{-x^{2} / 2} y^{\prime}(x / \sqrt{l s}) \mathrm{d} x\right. \\
& \left.-\sum_{l=1}^{\infty}(\alpha \sqrt{l} \rho R(\rho)-\rho / \sqrt{l s}) p(l s) \frac{e^{-\frac{1}{2} \alpha^{2} l}}{\sqrt{2 \pi}}\right],
\end{aligned}
$$

where

$$
R(\rho)=\frac{1-\rho}{\sqrt{\gamma}}=1-\frac{1}{3}(1-\rho)+\cdots .
$$

For the Gaussian random walk we have that (see [13])

$$
\begin{aligned}
\mathbb{E} M_{\beta} & =\sum_{l=1}^{\infty}\left(\frac{e^{-\frac{1}{2} \beta^{2} l}}{\sqrt{2 \pi l}}-\beta \frac{1}{\sqrt{2 \pi}} \int_{\beta \sqrt{l}}^{\infty} e^{-x^{2} / 2} \mathrm{~d} x\right), \\
\operatorname{Var} M_{\beta} & =\sum_{l=1}^{\infty}\left(\left(\beta^{2} l+1\right) \frac{1}{\sqrt{2 \pi}} \int_{\beta \sqrt{l}}^{\infty} e^{-x^{2} / 2} \mathrm{~d} x-\frac{\beta}{\sqrt{2 \pi}} l^{1 / 2} e^{-\frac{1}{2} \beta^{2} l}\right) .
\end{aligned}
$$

Ignoring the factors $\sqrt{s}$ and $s$, we again have complete agreement with the Gaussian random walk when $\lambda \rightarrow \infty$. The deviations from the Gaussian random walk are embodied by $p \not \equiv 1, y^{\prime} \not \equiv 1, \beta \not \equiv \alpha$ and the fact that $R(\rho) \not \equiv 1$ when $\rho<1$. The introduced notation is summarized in Table 1 .

\section{Results for $\boldsymbol{G}_{\boldsymbol{k}}$}

In this section we give a reformulation of the function $G_{k}$ in terms of a principal series expansion. The level of generality is higher than needed for the $\mathrm{M} / \mathrm{D} / \mathrm{s}$ queue, as we consider a large class of functions $z(x)$ of which $y^{\prime}(x)$ is just a special case. In Sect. 4.1 we derive the Taylor series for the most general case. We also discuss 
some special cases that lead to considerable reductions in complexity of the expressions. The principal series expansion comprises terms involving $s, z, a=\alpha / \sqrt{s}$ and elementary functions, as well as a constant $L_{k}$, not depending on $a=\alpha / \sqrt{s}$, which is more complicated. For this $L_{k}$ we present an asymptotic series as $s \rightarrow \infty$ that can be used conveniently when the radius of convergence $r_{0}$ of $z(x)=\sum_{j=0}^{\infty} b_{j} z^{j}$ is not small (for instance $2 \sqrt{\pi}$ as in the pilot case $z(x)=y^{\prime}(x)$ ). In Sect. 4.1 we derive the principal series expansion. In Sect. 4.2 we investigate the numerical evaluation of $L_{k}$ in terms of the optimal truncation value of the series expansions. In Sect. 4.3 we use this general result for the specific case of $z(x)=y^{\prime}(x)$ to derive series expansions and asymptotics for $\mathbb{P}\left(Q_{\lambda}=0\right)$. There is a clear connection to the Gaussian random walk. In fact, results for the Gaussian random walk involve the function $G_{k}$ for the special case $z(x) \equiv 1$.

\subsection{Principal series expansion}

We let $z:[0, \infty) \mapsto \mathbb{C}$ be a continuous function satisfying $z(x)=\mathcal{O}\left(\exp \left(\varepsilon x^{2}\right)\right)$ for any $\varepsilon>0$, and we assume that there is an $r_{0}>0$ such that $z(x)$ is represented by its Taylor series $\sum_{j=0}^{\infty} b_{j} x^{j}$ for $0 \leq x<r_{0}$. We consider for $s>0$ and integer $k$ the function

$$
G_{k}(a)=\sum_{l=1}^{\infty} l^{k+1 / 2} \int_{a}^{\infty} e^{-\frac{1}{2} l s x^{2}} z(x) \mathrm{d} x, \quad a>0 .
$$

In the case that $z(x)=x^{i}$ we have

$$
G_{k}(a)=s^{-\frac{i+1}{2}} T_{k, i}(a \sqrt{s}),
$$

where $T_{k, i}$ is defined as

$$
T_{k, i}(b)=\sum_{l=1}^{\infty} l^{k+1 / 2} \int_{b}^{\infty} e^{-\frac{1}{2} l x^{2}} x^{i} \mathrm{~d} x
$$

with $i=0,1, \ldots$ and $k \in \mathbb{Z}$. The functions $T_{k, i}$ have been thoroughly investigated in [14], Sect. 5, leading to analytic expressions. We now generalize this result to $G_{k}$.

Theorem 3 For $k \in \mathbb{Z}$ and $a<2 \sqrt{\pi / s}$ we have that

$$
\begin{aligned}
G_{k}(a)= & \left(\frac{2}{s}\right)^{k+3 / 2} \Gamma(k+3 / 2)\left\{\sum_{j=0}^{2 k+1} \frac{b_{j} a^{j-2 k-2}}{2 k+2-j}\right. \\
& \left.-b_{2 k+2} \ln a-\int_{0}^{a} \frac{z(x)-\sum_{j=0}^{2 k+2} b_{j} x^{j}}{x^{2 k+3}} \mathrm{~d} x\right\} \\
& +L_{k}-\sum_{r=0}^{\infty} \zeta(-k-r-1 / 2) \frac{\left(-\frac{1}{2} s\right)^{r}}{r !} \int_{0}^{a} x^{2 r} z(x) \mathrm{d} x,
\end{aligned}
$$


where

$$
\begin{aligned}
L_{k}= & \sum_{l=1}^{\infty} l^{k+1 / 2} \int_{0}^{\infty} e^{-\frac{1}{2} l s x^{2}}\left(z(x)-\sum_{j=0}^{2 k+2} b_{j} x^{j}\right) \mathrm{d} x \\
& +\frac{1}{2} \sum_{j=0}^{2 k+1} b_{j} \Gamma\left(\frac{j+1}{2}\right)\left(\frac{2}{s}\right)^{\frac{j+1}{2}} \zeta(-k+j / 2) \\
& +b_{2 k+2}\left(\frac{2}{s}\right)^{k+3 / 2} \Gamma(k+3 / 2)\left(\sum_{j=0}^{k} \frac{1}{2 j+1}-\ln \sqrt{2 s}\right) .
\end{aligned}
$$

Proof We have

$$
G_{k}^{\prime}(a)=-z(a) \sum_{l=1}^{\infty} l^{k+1 / 2} e^{-\frac{1}{2} l s a^{2}} .
$$

The right-hand side of (60) can be expressed in terms of Lerch's transcendent $\Phi$, defined as the analytic continuation of the series

$$
\Phi(z, t, v)=\sum_{n=0}^{\infty}(v+n)^{-t} z^{n}
$$

which converges for any real number $v \neq 0,-1,-2, \ldots$ if $z$ and $t$ are any complex numbers with either $|z|<1$, or $|z|=1$ and $\operatorname{Re}(t)>1$. Note that $\zeta(t)=\Phi(1, t, 1)$. Thus,

$$
G_{k}^{\prime}(a)=-z(a) e^{-\frac{1}{2} s a^{2}} \Phi\left(e^{-\frac{1}{2} s a^{2}},-k-\frac{1}{2}, 1\right) .
$$

We then use the important result derived by Bateman [9], Sect. 1.11(8) (with $\zeta(t, v):=\Phi(1, t, v)$ the Hurwitz zeta function)

$$
\Phi(z, t, v)=\frac{\Gamma(1-t)}{z^{v}}(\ln 1 / z)^{t-1}+z^{-v} \sum_{r=0}^{\infty} \zeta(t-r, v) \frac{(\ln z)^{r}}{r !}
$$

which holds for $|\ln z|<2 \pi, t \neq 1,2,3, \ldots$, and $v \neq 0,-1,-2, \ldots$, as to obtain

$$
G_{k}^{\prime}(a)=-z(a)\left[\Gamma(k+3 / 2)\left(\frac{2}{s}\right)^{k+3 / 2} a^{-2 k-3}+\sum_{r=0}^{\infty} \zeta(-k-r-1 / 2) \frac{\left(-\frac{1}{2} s a^{2}\right)^{r}}{r !}\right]
$$

Therefore

$$
G_{k}^{\prime}(a)+\Gamma(k+3 / 2)\left(\frac{2}{s}\right)^{k+3 / 2} \sum_{j=0}^{2 k+2} b_{j} a^{j-2 k-3}
$$




$$
\begin{aligned}
= & -\Gamma(k+3 / 2)\left(\frac{2}{s}\right)^{k+3 / 2}\left(z(a)-\sum_{j=0}^{2 k+2} b_{j} a^{j}\right) a^{-2 k-3} \\
& -z(a) \sum_{r=0}^{\infty} \zeta(-k-r-1 / 2) \frac{\left(-\frac{1}{2} s a^{2}\right)^{r}}{r !} .
\end{aligned}
$$

The series on the second line of (65) converges uniformly in $a$ with $s^{1 / 2} a \in[0, c]$ and $c<2 \sqrt{\pi}$, so upon integrating the identity in (65) we get for $\frac{1}{2} s a^{2} \leq c<2 \pi$

$$
\begin{aligned}
& G_{k}(a)+\Gamma(k+3 / 2)\left(\frac{2}{s}\right)^{k+3 / 2}\left\{\sum_{j=0}^{2 k+1} \frac{b_{j} a^{j-2 k-2}}{j-2 k-2}+b_{2 k+2} \ln a\right\} \\
& =L_{k}-\Gamma(k+3 / 2)\left(\frac{2}{s}\right)^{k+3 / 2} \int_{0}^{a}\left(z(x)-\sum_{j=0}^{2 k+2} b_{j} x^{j}\right) x^{-2 k-3} \mathrm{~d} x \\
& -\sum_{r=0}^{\infty} \zeta(-k-r-1 / 2) \frac{\left(-\frac{1}{2} s\right)^{r}}{r !} \int_{0}^{a} x^{2 r} z(x) \mathrm{d} x,
\end{aligned}
$$

where

$$
L_{k}=\lim _{a \downarrow 0}\left[G_{k}(a)+\Gamma(k+3 / 2)\left(\frac{2}{s}\right)^{k+3 / 2}\left\{\sum_{j=0}^{2 k+1} \frac{b_{j} a^{j-2 k-2}}{j-2 k-2}+b_{2 k+2} \ln a\right\}\right] .
$$

We shall determine $L_{k}$. It holds that, as $a \downarrow 0$,

$$
\begin{aligned}
G_{k}(a)= & \sum_{l=1}^{\infty} l^{k+1 / 2} \int_{a}^{\infty} e^{-\frac{1}{2} l s x^{2}}\left(z(x)-\sum_{i=0}^{2 k+2} b_{i} x^{i}\right) \mathrm{d} x \\
& +\sum_{i=0}^{2 k+2} b_{i} \sum_{l=1}^{\infty} l^{k+1 / 2} \int_{a}^{\infty} e^{-\frac{1}{2} l s x^{2}} x^{i} \mathrm{~d} x \\
= & \sum_{l=1}^{\infty} l^{k+1 / 2} \int_{0}^{\infty} e^{-\frac{1}{2} l s x^{2}}\left(z(x)-\sum_{i=0}^{2 k+2} b_{i} x^{i}\right) \mathrm{d} x \\
& +o(1)+\sum_{i=0}^{2 k+2} b_{i} s^{-\frac{i+1}{2}} T_{k, i}(a \sqrt{s}) .
\end{aligned}
$$

Here (56) has been used and the $o(1)$ comes from the fact that $z(x)-\sum_{i=0}^{2 k+2} b_{i} x^{i}=$ $\mathcal{O}\left(x^{2 k+3}\right)$ so that

$$
\int_{0}^{a} e^{-\frac{1}{2} l s x^{2}}\left(z(x)-\sum_{i=0}^{2 k+2} b_{i} x^{i}\right) \mathrm{d} x=\mathcal{O}\left(\frac{1}{(l s)^{k+2}}\right)
$$


Now from Janssen and Van Leeuwaarden [14], Sect. 5,

$$
\begin{aligned}
s^{-\frac{i+1}{2}} T_{k, i}(a \sqrt{s}) & =s^{-\frac{i+1}{2}}\left[\frac{\Gamma(k+3 / 2)}{2 k+2-i} 2^{k+3 / 2}(a \sqrt{s})^{i-2 k-2}+L_{k, i}+\mathcal{O}(a)\right] \\
& =\Gamma(k+3 / 2)\left(\frac{2}{s}\right)^{k+3 / 2} \frac{a^{i-2 k-2}}{2 k+2-i}+s^{-\frac{i+1}{2}} L_{k, i}+\mathcal{O}(a)
\end{aligned}
$$

for $i=0,1, \ldots, 2 k+1$ and

$$
\begin{aligned}
s^{-\frac{2 k+3}{2}} T_{k, 2 k+2}(a \sqrt{s})= & s^{-\frac{2 k+3}{2}}\left[-\Gamma(k+3 / 2) 2^{k+3 / 2} \ln (a \sqrt{s})+L_{k, 2 k+2}+\mathcal{O}(a)\right] \\
= & -\Gamma(k+3 / 2)\left(\frac{2}{s}\right)^{k+3 / 2} \ln (a \sqrt{s}) \\
& +s^{-(k+3 / 2)} L_{k, 2 k+2}+\mathcal{O}(a) .
\end{aligned}
$$

Here

$$
\begin{aligned}
L_{k, i} & =\frac{1}{2} \Gamma\left(\frac{i+1}{2}\right) 2^{\frac{i+1}{2}} \zeta(-k+i / 2), \quad i=0,1, \ldots, 2 k+1, \\
L_{k, 2 k+2} & =\Gamma(k+3 / 2) 2^{k+3 / 2}\left(\sum_{j=0}^{k} \frac{1}{2 j+1}-\frac{1}{2} \ln 2\right) .
\end{aligned}
$$

Therefore, as $a \downarrow 0$, we get

$$
\begin{aligned}
G_{k}(a)= & \sum_{l=1}^{\infty} l^{k+1 / 2} \int_{0}^{\infty} e^{-\frac{1}{2} l s x^{2}}\left(z(x)-\sum_{i=0}^{2 k+2} b_{i} x^{i}\right) \mathrm{d} x+o(1) \\
& +\sum_{i=0}^{2 k+1} b_{i}\left[\Gamma(k+3 / 2)\left(\frac{2}{s}\right)^{k+3 / 2} \frac{a^{i-2 k-2}}{2 k+2-i}\right. \\
& \left.+\frac{1}{2} \Gamma\left(\frac{i+1}{2}\right)\left(\frac{2}{s}\right)^{\frac{i+1}{2}} \zeta(-k+i / 2)\right] \\
& +b_{2 k+2} \Gamma(k+3 / 2)\left(\frac{2}{s}\right)^{k+3 / 2} \\
& \times\left[-\ln a-\ln \sqrt{s}+\sum_{j=0}^{k} \frac{1}{2 j+1}-\ln \sqrt{2}\right] .
\end{aligned}
$$

Adding

$$
\Gamma(k+3 / 2)\left(\frac{2}{s}\right)^{k+3 / 2}\left\{\sum_{j=0}^{2 k+1} \frac{b_{j} a^{j-2 k-2}}{j-2 k-2}+b_{2 k+2} \ln a\right\}
$$


Table 2 Some values of the Riemann zeta function $\zeta$

\begin{tabular}{llll}
\hline$x$ & $\zeta(x)$ & $x$ & $\zeta(x)$ \\
\hline-5.5 & -0.00267145801990 & 0.0 & -0.50000000000000 \\
-5.0 & -0.00396825396825 & 0.5 & -1.46035450880959 \\
-4.5 & -0.00309166924722 & 1.5 & 2.61237534868549 \\
-4.0 & 0 & 2.0 & 1.64493406684823 \\
-3.5 & 0.00444101133548 & 2.5 & 1.34148725725092 \\
-3.0 & 0.00833333333333 & 3.0 & 1.20205690315959 \\
-2.5 & 0.00851692877785 & 3.5 & 1.12673386731706 \\
-2.0 & 0 & 4.0 & 1.08232323371114 \\
-1.5 & -0.02548520188983 & 4.5 & 1.05470751076145 \\
-1.0 & -0.08333333333333 & 5.0 & 1.03692775514337 \\
-0.5 & -0.20788622497735 & 5.5 & 1.02520457995469 \\
\hline
\end{tabular}

at either side of (74) and letting $a \downarrow 0$, we find that $L_{k}$ has the required value (59). Then (58) follows from (66).

Some values of the Riemann zeta function $\zeta$ are given in Table 2 .

We now give several special cases of Theorem 3 . The next two corollaries focus on negative values of $k$.

Corollary 1 For $\frac{1}{2} s a^{2}<2 \pi$ and $k=-2,-3, \ldots$ we have that

$$
\begin{aligned}
G_{k}(a)= & -\left(\frac{2}{s}\right)^{k+3 / 2} \Gamma(k+3 / 2) \int_{0}^{a} x^{-2 k-3} z(x) \mathrm{d} x \\
& +L_{k}-\sum_{r=0}^{\infty} \zeta(-k-r-1 / 2) \frac{\left(-\frac{1}{2} s\right)^{r}}{r !} \int_{0}^{a} x^{2 r} z(x) \mathrm{d} x,
\end{aligned}
$$

where $L_{k}=\sum_{l=1}^{\infty} l^{k+1 / 2} \int_{0}^{\infty} e^{-\frac{1}{2} l s x^{2}} z(x) \mathrm{d} x$ (which follows from the definition of $L_{k}$ in (59) in which all series over $j$ vanish for $k=-2,-3, \ldots)$.

Corollary 2 For $\frac{1}{2} s a^{2}<2 \pi$ and $k=-1$ we have that

$$
\begin{aligned}
G_{-1}(a)= & -\left(\frac{2 \pi}{s}\right)^{1 / 2}\left\{\ln a+\int_{0}^{a} \frac{z(x)-b_{0}}{x} \mathrm{~d} x\right\} \\
& +L_{-1}-\sum_{r=0}^{\infty} \zeta(-r+1 / 2) \frac{\left(-\frac{1}{2} s\right)^{r}}{r !} \int_{0}^{a} x^{2 r} z(x) \mathrm{d} x,
\end{aligned}
$$

where $L_{-1}=\sum_{l=1}^{\infty} l^{-1 / 2} \int_{0}^{\infty} e^{-\frac{1}{2} l s x^{2}}\left(z(x)-b_{0}\right) \mathrm{dx}-\left(\frac{2 \pi}{s}\right)^{1 / 2} \ln \sqrt{2 s}$.

Theorem 3 is meant for the case that $a$ and the convergence radius $r_{0}$ of $\sum_{j=0}^{\infty} b_{j} x^{j}$ are general. In the case that $a<r_{0}$ the expressions can be simplified 
considerably, as demonstrated below. If $a<r_{0}$ we have

$$
\begin{aligned}
\int_{0}^{a} \frac{z(x)-\sum_{j=0}^{2 k+2} b_{j} x^{j}}{x^{2 k+3}} \mathrm{~d} x & =\sum_{j=2 k+3}^{\infty} \frac{b_{j} a^{j-2 k-2}}{j-2 k-2}, \\
\int_{0}^{a} x^{2 r} z(x) \mathrm{d} x & =\sum_{j=0}^{\infty} \frac{b_{j} a^{j+2 r+1}}{j+2 r+1} .
\end{aligned}
$$

As a consequence of (78) we have that the expression on the first line of (58)

$$
\sum_{j=0}^{2 k+1} \frac{b_{j} a^{j-2 k-2}}{2 k+2-j}-b_{2 k+2} \ln a-\int_{0}^{a} \frac{z(x)-\sum_{j=0}^{2 k+2} b_{j} x^{j}}{x^{2 k+3}} \mathrm{~d} x
$$

simplifies to

$$
\sum_{j=0, j \neq 2 k+2}^{\infty} \frac{b_{j} a^{j-2 k-2}}{2 k+2-j}-b_{2 k+2} \ln a .
$$

Together with (79) this gives expressions for $G_{k}(a)$ that are, apart from the $L_{k}$ to which we turn next, convenient for computation when $a$ is small.

Lemma 3 For the first line of (59)

$$
\begin{aligned}
& \sum_{l=1}^{\infty} l^{k+1 / 2} \int_{0}^{\infty} e^{-\frac{1}{2} l s x^{2}}\left(z(x)-\sum_{j=0}^{2 k+2} b_{j} x^{j}\right) \mathrm{d} x \\
& +\frac{1}{2} \sum_{j=0}^{2 k+1} b_{j} \Gamma\left(\frac{j+1}{2}\right)\left(\frac{2}{s}\right)^{\frac{j+1}{2}} \zeta(-k+j / 2)
\end{aligned}
$$

there is the asymptotic expression

$$
\sim \frac{1}{2} \sum_{j=0, j \neq 2 k+2}^{\infty} b_{j} \Gamma\left(\frac{j+1}{2}\right)\left(\frac{2}{s}\right)^{\frac{j+1}{2}} \zeta(-k+j / 2), \quad s \rightarrow \infty .
$$

In case that $b_{j} \Gamma\left(\frac{j+1}{2}\right)=\mathcal{O}\left(B^{j}\right)$ for some $B>0$, the asymptotic series in (83) is convergent when $s>2 B^{2}$, with sum equal to (82).

Proof Using

$$
\begin{aligned}
\int_{0}^{\infty} e^{-\frac{1}{2} l s x^{2}} x^{j} \mathrm{~d} x & =\frac{1}{2}\left(\frac{2}{l s}\right)^{(j+1) / 2} \int_{0}^{\infty} e^{-u} u^{(j-1) / 2} \mathrm{du} \\
& =\frac{1}{2}\left(\frac{2}{l s}\right)^{(j+1) / 2} \Gamma\left(\frac{j+1}{2}\right)
\end{aligned}
$$


we find that

$$
\begin{aligned}
& \sum_{l=1}^{\infty} l^{k+1 / 2} \int_{0}^{\infty} e^{-\frac{1}{2} l s x^{2}}\left(z(x)-\sum_{j=0}^{2 k+2} b_{j} x^{j}\right) \mathrm{d} x \\
& \quad \sim \sum_{j=2 k+3}^{\infty} b_{j} \sum_{l=1}^{\infty} l^{k+1 / 2} \int_{0}^{\infty} e^{-\frac{1}{2} l s x^{2}} x^{j} \mathrm{~d} x \\
& \quad=\frac{1}{2} \sum_{j=2 k+3}^{\infty} b_{j}\left(\frac{2}{s}\right)^{(j+1) / 2} \Gamma\left(\frac{j+1}{2}\right) \sum_{l=1}^{\infty} l^{-j / 2+k}
\end{aligned}
$$

This yields $(83)$ since $\zeta(j / 2-k)=\sum_{l=1}^{\infty} l^{-j / 2+k}$.

Remark 4 The series expansion (58) for $G_{k}(a)$ comprises, as $a \downarrow 0$, leading order terms involving $a^{j-2 k-2}, j=0,1, \ldots, 2 k+1$, and $\ln a$ when $k=-1,0,1, \ldots$, and $G_{k}(a)$ stays bounded as $a \downarrow 0$ for $k=-2,-3,-4, \ldots$ In most cases we are interested in, the value of $a$ is quite small (say $\leq 0.1$ ). The formula in (58) can be used conveniently for computation of $G_{k}(a)$ for values of $a$ from 0 to as large as $\sqrt{\pi}$. For larger values of $a$, we present in the appendix formula (165) as an attractive alternative to compute $G_{k}(a)$. This alternative shows, for instance, quite clearly an $\exp \left(-\frac{1}{2}(s+1) a^{2}\right)$-behavior of $G_{k}(a)$ as $a$ gets large.

Remark 5 Chang and Peres [6], Theorem 1.1, proved that

$$
\mathbb{P}\left(M_{\beta}=0\right)=\sqrt{2} \beta \exp \left\{\frac{\beta}{\sqrt{2 \pi}} \sum_{r=0}^{\infty} \frac{\zeta(1 / 2-r)}{r !(2 r+1)}\left(\frac{-\beta^{2}}{2}\right)^{r}\right\},
$$

for $0<\beta<2 \sqrt{\pi}$. This result follows from Theorem 3 , for the case $z(x) \equiv 1, a=$ $\beta / \sqrt{s}$ and $k=-1$.

For general $k$, setting $z(x) \equiv 1$ and $a=\beta / \sqrt{s}$ in Theorem 3 leads to the following result.

Lemma 4 For $\beta<2 \sqrt{\pi}$ and $k \in \mathbb{Z}$ we have that

$$
\sum_{l=1}^{\infty} l^{k} P(-\beta \sqrt{l})=-\frac{\beta}{\sqrt{2 \pi}} \sum_{r=0}^{\infty} \frac{\zeta\left(-k-r-\frac{1}{2}\right)}{r !(2 r+1)}\left(\frac{-\beta^{2}}{2}\right)^{r}+R_{k}(\beta),
$$

where $R_{-1}(\beta)=-\ln \sqrt{2} \beta$ and

$$
R_{k}(\beta)=\frac{1}{\sqrt{2 \pi}} \frac{\Gamma\left(k+\frac{3}{2}\right)}{2 k+2} 2^{k+\frac{3}{2}} \beta^{-2 k-2}+\frac{1}{2} \zeta(-k), \quad k \neq-1 .
$$




\subsection{Optimal truncation value}

Lemma 3 can be deployed in two ways. We can take only the first few terms to get a good idea of how things behave (see Sect. 4.3), or for the numerical evaluation of $L_{k}$, we take as many terms as needed using optimal truncation. The optimal truncation value $J$ of (83) is so large (see developments below) that we can replace $\zeta(-k+J / 2)$ by 1 . The truncation error made by approximating (82) by

$$
\frac{1}{2} \sum_{j=0, j \neq 2 k+2}^{J} b_{j} \Gamma\left(\frac{j+1}{2}\right)\left(\frac{2}{s}\right)^{(j+1) / 2} \zeta(-k+j / 2)
$$

is of the order

$$
\frac{1}{2} b_{J+1} \Gamma\left(\frac{J+2}{2}\right)\left(\frac{2}{s}\right)^{(J+2) / 2} .
$$

We replace, furthermore, $b_{J+1}=(J+2) a_{J+2}$ by its asymptotic bound, see the appendix, Lemma 13,

$$
\left|b_{J+1}\right| \leq\left(\frac{J+2}{2}\right)^{-1 / 2}\left(\frac{1}{2 \sqrt{\pi}}\right)^{J+2}
$$

Thus

$$
\left|\frac{1}{2} b_{J+1} \Gamma\left(\frac{J+2}{2}\right)\left(\frac{2}{s}\right)^{\frac{J+2}{2}}\right| \leq\left(\frac{1}{2(J+2)}\right)^{1 / 2} \Gamma\left(\frac{J+2}{2}\right)\left(\frac{1}{2 \pi s}\right)^{(J+2) / 2} .
$$

The factor $(1 / 2(J+2))^{1 / 2}$ is rather unimportant for determination of the optimal truncation value $J$, and we focus on

$$
D_{J}=\Gamma\left(\frac{J+2}{2}\right)\left(\frac{1}{2 \pi s}\right)^{(J+2) / 2} .
$$

Noting that $\Gamma(J / 2+3 / 2) / \Gamma(J / 2+1) \approx(J / 2+1)^{1 / 2}$, we see that

$$
\frac{D_{J+1}}{D_{J}} \approx\left(\frac{J+2}{2}\right)^{1 / 2}\left(\frac{1}{2 \pi s}\right)^{1 / 2}
$$

The right-hand side of (94) decreases in $J$ until $J / 2+1=2 \pi s$; this $J$ is (near to) the optimal truncation point. At this point we estimate the right-hand side of (92) by Stirling's formula as

$$
\begin{aligned}
\left(\frac{1}{8 \pi s}\right)^{1 / 2} \Gamma(2 \pi s)\left(\frac{1}{2 \pi s}\right)^{2 \pi s} & \approx\left(\frac{1}{8 \pi s}\right)^{1 / 2}(2 \pi s)^{2 \pi s-1 / 2} e^{-2 \pi s} \sqrt{2 \pi}\left(\frac{1}{2 \pi s}\right)^{2 \pi s} \\
& =\frac{e^{-2 \pi s}}{s \sqrt{8 \pi}}
\end{aligned}
$$

For instance, for $s=10$ this equals $10^{-29}$. 
Remark 6 Observe how important it is that we have managed to show the good bound (91) on $\left|b_{J+1}\right|$. If, for instance, the $1 / 2 \sqrt{\pi}$ in this bound were to be replaced by 1 , the $e^{-2 \pi s}$ on the far right of (95) would have to be replaced by $e^{-s / 2}$ and the resulting quantity $\sqrt{2 \pi} e^{-s / 2} / s$ would be 0.0017 for $s=10$.

\subsection{Accurate approximations for the $\mathrm{M} / \mathrm{D} / s$ queue}

We can apply Theorem 3 to obtain accurate approximations for the emptiness probability and the mean and variance of the queue length. By way of illustration, we do this in some detail for $\mathbb{P}\left(Q_{\lambda}=0\right)$ and briefly indicate at the end of this section how one can proceed for the other cases.

We have from (27) and (29) that

$$
\begin{aligned}
-\ln \mathbb{P}\left(Q_{\lambda}=0\right) \sim & \frac{1}{\sqrt{2 \pi}} \sum_{k=0}^{\infty} p_{k} s^{-k+1 / 2} G_{-(k+1)}(\alpha / \sqrt{s}) \\
= & \frac{1}{\sqrt{2 \pi}}\left(s^{1 / 2} G_{-1}(\alpha / \sqrt{s})-\frac{1}{12} s^{-1 / 2} G_{-2}(\alpha / \sqrt{s})\right. \\
& \left.+\frac{1}{288} s^{-3 / 2} G_{-3}(\alpha / \sqrt{s})+\cdots\right) .
\end{aligned}
$$

The $G_{-2}, G_{-3}, \ldots$ are bounded functions of $a=\alpha / \sqrt{s}$ while $G_{-1}(a)$ behaves like

$$
-\left(\frac{2 \pi}{s}\right)^{1 / 2} \ln a \sqrt{2 s} \quad \text { as } a \downarrow 0 .
$$

Accurate approximations to $-\ln \mathbb{P}\left(Q_{\lambda}=0\right)$ are obtained by including $1,2,3, \ldots$ terms of the second line of (96) in which the $G$ 's must be approximated. For the number of terms of the asymptotic series in (96) to be included one could follow a truncation strategy (based on (141), (154) and the bound $G_{-k}(a) \leq\left(\frac{\pi}{2 s}\right)^{1 / 2} \zeta(k)$, $k=2,3, \ldots)$ pretty much as was done in Sect. 4.2 . We shall not pursue this point here.

We shall compute accurate approximations to $G_{-k}(a)$ for $k=1,2, \ldots$ We have from (76) and (77) for $\alpha<2 \sqrt{\pi}$

$$
\begin{aligned}
G_{-1}(\alpha / \sqrt{s})= & -\left(\frac{2 \pi}{s}\right)^{1 / 2}\left\{\ln \alpha / \sqrt{s}+\int_{0}^{\alpha / \sqrt{s}} \frac{y^{\prime}(x)-1}{x} \mathrm{~d} x\right\} \\
& +L_{-1}-\sum_{r=0}^{\infty} \zeta\left(-r+\frac{1}{2}\right) \frac{\left(-\frac{1}{2} s\right)^{r}}{r !} \int_{0}^{\alpha / \sqrt{s}} x^{2 r} y^{\prime}(x) \mathrm{d} x,
\end{aligned}
$$

and for $k=2,3, \ldots$,

$$
\begin{aligned}
G_{-k}(\alpha / \sqrt{s})= & -\left(\frac{2}{s}\right)^{-k+3 / 2} \Gamma(-k+3 / 2) \int_{0}^{\alpha / \sqrt{s}} x^{2 k-3} y^{\prime}(x) \mathrm{d} x \\
& +L_{-k}-\sum_{r=0}^{\infty} \zeta\left(k-r-\frac{1}{2}\right) \frac{\left(-\frac{1}{2} s\right)^{r}}{r !} \int_{0}^{\alpha / \sqrt{s}} x^{2 r} y^{\prime}(x) \mathrm{d} x .
\end{aligned}
$$


Here,

$$
L_{-1}=\sum_{l=1}^{\infty} l^{-1 / 2} \int_{0}^{\infty} e^{-\frac{1}{2} l s x^{2}}\left(y^{\prime}(x)-1\right) \mathrm{dx}-\left(\frac{2 \pi}{s}\right)^{1 / 2} \ln \sqrt{2 s}
$$

and for $k=2,3, \ldots$,

$$
L_{-k}=\sum_{l=1}^{\infty} l^{-k+1 / 2} \int_{0}^{\infty} e^{-\frac{1}{2} l s x^{2}} y^{\prime}(x) \mathrm{d} x .
$$

Below we specify the missing ingredients in (98)-(101).

- We have

$$
\int_{0}^{\alpha / \sqrt{s}} \frac{y^{\prime}(x)-1}{x} \mathrm{~d} x=\int_{0}^{\alpha / \sqrt{s}} \sum_{j=1}^{\infty} b_{j} x^{j-1} \mathrm{~d} x=\sum_{j=1}^{\infty} \frac{b_{j}}{j}\left(\frac{\alpha}{\sqrt{s}}\right)^{j},
$$

and the computation of the series is feasible when $0 \leq \alpha / \sqrt{s} \leq 2 \sqrt{\pi}$, the $b_{j}$ being computable and $\mathcal{O}\left(1 /(2 \sqrt{\pi})^{j}\right)$.

- We have

$$
\int_{0}^{\alpha / \sqrt{s}} x^{n} y^{\prime}(x) \mathrm{d} x=\int_{0}^{\alpha / \sqrt{s}} \sum_{j=0}^{\infty} b_{j} x^{n+j} \mathrm{~d} x=\sum_{j=0}^{\infty} \frac{b_{j}}{n+j+1}\left(\frac{\alpha}{\sqrt{s}}\right)^{n+j+1},
$$

and the computation of the series is feasible when $0 \leq \alpha / \sqrt{s} \leq 2 \sqrt{\pi}$. Furthermore

$$
\left(-\frac{1}{2} s\right)^{r} \int_{0}^{\alpha / \sqrt{s}} x^{2 r} y^{\prime}(x) \mathrm{d} x=\left(-\frac{1}{2} \alpha^{2}\right)^{r} \sum_{j=0}^{\infty} \frac{b_{j}}{2 r+j+1}\left(\frac{\alpha}{\sqrt{s}}\right)^{j+1}
$$

Since, see [13], Sect. 6, $\zeta\left(-r+\frac{1}{2}\right) / r !=\mathcal{O}\left(1 /(2 \pi)^{r}\right)$, the computation of the series over $r$ at the right-hand side of (98) is feasible when $\alpha<2 \sqrt{\pi}$. A similar result holds for the series over $r$ at the right-hand side of (99).

- We have by Lemma 3

$$
\begin{aligned}
& \sum_{l=1}^{\infty} l^{-1 / 2} \int_{0}^{\infty} e^{-\frac{1}{2} l s x^{2}}\left(y^{\prime}(x)-1\right) \mathrm{d} x \\
& \quad \sim \frac{1}{2} \sum_{j=1}^{\infty} b_{j} \Gamma\left(\frac{j+1}{2}\right)\left(\frac{2}{s}\right)^{\frac{j+1}{2}} \zeta(1+j / 2), \\
& L_{-k} \sim \frac{1}{2} \sum_{j=0}^{\infty} b_{j} \Gamma\left(\frac{j+1}{2}\right)\left(\frac{2}{s}\right)^{\frac{j+1}{2}} \zeta(k+j / 2), \quad k=2,3, \ldots,
\end{aligned}
$$

for the series expressions at the right-hand sides of (100) and (101). The left-hand sides of (105) and (106) can be accurately approximated by using the optimal truncation approach of Sect. 4.2. Alternatively, assume that we include all three terms 
on the second line of (96) (so that the truncation error is $\mathcal{O}\left(s^{-7 / 2}\right)$ ). We then include in the right-hand side of (105) the terms with $j=1,2,3,4$, and in the righthand side of (106) the terms with $j=1,2$.

When we want to compute accurate approximations to $\mathbb{E} Q_{\lambda}$ and $\operatorname{Var} Q_{\lambda}$, we can use (50) and (51), and then it becomes necessary to approximate $G_{k}(a)$ with $k=0$ and $k=1$ as well. This can still be done by using Theorem 3 with its simplifications as pointed out in Corollary 2 since $z(x)=y^{\prime}(x)$ has $b_{j}=\mathcal{O}\left((2 \sqrt{\pi})^{-j}\right)$. Of course, there are various ways to proceed here, just like in case of $-\ln \mathbb{P}\left(Q_{\lambda}=0\right)$ treated above. For the latter case, we have just worked out one of the more straightforward methods.

Table 3 displays approximations to $\mathbb{P}\left(Q_{\lambda}=0\right)$ based on the series expansion (96). Results are given for 1, 2, and 3 terms of the second line of (96), and the $G$ 's are approximated as described in this subsection. Clearly, the expansions provide sharp approximations, and in most cases, one term suffices to get accurate results, i.e.,

$$
\mathbb{P}\left(Q_{\lambda}=0\right) \approx \exp \left\{-\frac{\sqrt{s}}{\sqrt{2 \pi}} G_{-1}(\alpha / \sqrt{s})\right\} .
$$

\section{Bounds and approximations for the emptiness probability}

The Gaussian form (24) for $\mathbb{P}\left(Q_{\lambda}=0\right)$ is rather complicated due to the presence of $p(l s)$ and $z(x)=y^{\prime}(x)$, which both can be expressed as infinite series. In this section we obtain bounds on $\mathbb{P}\left(Q_{\lambda}=0\right)$ by using inequalities for $p(l s)$ and $y^{\prime}(x)$.

\section{Lemma 5}

$$
\begin{aligned}
\mathbb{P}\left(Q_{\lambda}=0\right) & \geq \exp \left\{-s^{1 / 2} \sum_{l=1}^{\infty} \frac{1}{\sqrt{2 \pi l}} \int_{\sqrt{\gamma}}^{\infty} e^{-\frac{1}{2} l s x^{2}} y^{\prime}(x) \mathrm{d} x\right\}=: L B, \\
\mathbb{P}\left(Q_{\lambda}=0\right) & \leq \exp \left\{-s^{1 / 2} \sum_{l=1}^{\infty} \frac{1}{\sqrt{2 \pi l}}\left(1-\frac{1}{12 l s}\right) \int_{\sqrt{\gamma}}^{\infty} e^{-\frac{1}{2} l s x^{2}} y^{\prime}(x) \mathrm{d} x\right\} \\
& =: U B .
\end{aligned}
$$

Proof Follows directly from rewriting (24) as

$$
\mathbb{P}\left(Q_{\lambda}=0\right)=\exp \left\{-s^{1 / 2} \sum_{l=1}^{\infty} \frac{p(l s)}{\sqrt{2 \pi l}} \int_{\sqrt{\gamma}}^{\infty} e^{-\frac{1}{2} l s x^{2}} y^{\prime}(x) \mathrm{d} x\right\}
$$

and applying

$$
n^{-n-1 / 2} \frac{e^{n}}{\sqrt{2 \pi}}\left(1-\frac{1}{12 n}\right) \leq \frac{1}{n !} \leq n^{-n-1 / 2} \frac{e^{n}}{\sqrt{2 \pi}} .
$$


Table 3 Series expansions for $\mathbb{P}\left(Q_{\lambda}=0\right)$ based on (96). The values of $\mathbb{P}\left(M_{\beta}=0\right)$ are given in parentheses

\begin{tabular}{|c|c|c|c|c|c|c|c|c|c|c|}
\hline \multirow[t]{2}{*}{$s$} & \multirow[t]{2}{*}{$\alpha$} & \multicolumn{4}{|c|}{$\beta=0.01(0.0141)$} & \multirow[t]{2}{*}{$\alpha$} & \multicolumn{4}{|c|}{$\beta=0.1(0.1334)$} \\
\hline & & True & (96)-1 & $(96)-2$ & $(96)-3$ & & True & (96)-1 & $(96)-2$ & $(96)-3$ \\
\hline 1 & 0.0100 & 0.0268 & 0.0256 & 0.0267 & 0.0267 & 0.0983 & 0.2351 & 0.2265 & 0.2345 & 0.2343 \\
\hline 2 & 0.0100 & 0.0225 & 0.0219 & 0.0225 & 0.0225 & 0.0988 & 0.2022 & 0.1980 & 0.2021 & 0.2021 \\
\hline 5 & 0.0100 & 0.0190 & 0.0188 & 0.0190 & 0.0190 & 0.0993 & 0.1747 & 0.1730 & 0.1747 & 0.1746 \\
\hline 10 & 0.0100 & 0.0174 & 0.0173 & 0.0174 & 0.0174 & 0.0995 & 0.1617 & 0.1609 & 0.1617 & 0.1617 \\
\hline 20 & 0.0100 & 0.0164 & 0.0163 & 0.0164 & 0.0164 & 0.0996 & 0.1529 & 0.1525 & 0.1529 & 0.1529 \\
\hline 50 & 0.0100 & 0.0155 & 0.0155 & 0.0155 & 0.0155 & 0.0998 & 0.1455 & 0.1453 & 0.1455 & 0.1455 \\
\hline 100 & 0.0100 & 0.0151 & 0.0150 & 0.0151 & 0.0151 & 0.0998 & 0.1419 & 0.1418 & 0.1419 & 0.1419 \\
\hline 200 & 0.0100 & 0.0148 & 0.0148 & 0.0148 & 0.0148 & 0.0999 & 0.1393 & 0.1393 & 0.1393 & 0.1393 \\
\hline 500 & 0.0100 & 0.0145 & 0.0145 & 0.0145 & 0.0145 & 0.0999 & 0.1371 & 0.1371 & 0.1371 & 0.1371 \\
\hline \multirow[t]{2}{*}{$s$} & \multirow[t]{2}{*}{$\alpha$} & \multicolumn{4}{|c|}{$\beta=0.2(0.2518)$} & \multirow[t]{2}{*}{$\alpha$} & \multicolumn{4}{|c|}{$\beta=0.5(0.5293)$} \\
\hline & & True & (96)-1 & $(96)-2$ & $(96)-3$ & & True & $(96)-1$ & $(96)-2$ & (96)-3 \\
\hline 1 & 0.1932 & 0.4105 & 0.3979 & 0.4092 & 0.4089 & 0.4573 & 0.7182 & 0.7049 & 0.7137 & 0.7134 \\
\hline 2 & 0.1952 & 0.3613 & 0.3549 & 0.3611 & 0.3610 & 0.4699 & 0.6656 & 0.6586 & 0.6642 & 0.6641 \\
\hline 5 & 0.1970 & 0.3185 & 0.3159 & 0.3185 & 0.3185 & 0.4811 & 0.6156 & 0.6125 & 0.6151 & 0.6151 \\
\hline 10 & 0.1979 & 0.2979 & 0.2966 & 0.2979 & 0.2978 & 0.4867 & 0.5899 & 0.5883 & 0.5897 & 0.5897 \\
\hline 20 & 0.1985 & 0.2838 & 0.2831 & 0.2837 & 0.2837 & 0.4906 & 0.5719 & 0.5710 & 0.5717 & 0.5717 \\
\hline 50 & 0.1991 & 0.2716 & 0.2714 & 0.2716 & 0.2716 & 0.4941 & 0.5560 & 0.5557 & 0.5560 & 0.5560 \\
\hline 100 & 0.1993 & 0.2657 & 0.2655 & 0.2657 & 0.2657 & 0.4958 & 0.5481 & 0.5479 & 0.5481 & 0.5481 \\
\hline 200 & 0.1995 & 0.2615 & 0.2615 & 0.2615 & 0.2615 & 0.4970 & 0.5426 & 0.5425 & 0.5425 & 0.5425 \\
\hline 500 & 0.1997 & 0.2579 & 0.2579 & 0.2579 & 0.2579 & 0.4981 & 0.5377 & 0.5376 & 0.5377 & 0.5377 \\
\hline \multirow[t]{2}{*}{$s$} & \multirow[t]{2}{*}{$\alpha$} & \multicolumn{4}{|c|}{$\beta=1(0.8005)$} & $\alpha$ & \multicolumn{4}{|c|}{$\beta=2(0.9762)$} \\
\hline & & True & (96)-1 & (96)-2 & $(96)-3$ & & True & $(96)-1$ & $(96)-2$ & (96)-3 \\
\hline 1 & 0.8299 & 0.9055 & 0.8973 & 0.8948 & 0.8945 & 1.3670 & 0.9835 & 0.9793 & 0.9636 & 0.9633 \\
\hline 2 & 0.8790 & 0.8787 & 0.8746 & 0.8737 & 0.8736 & 1.5296 & 0.9799 & 0.9787 & 0.9674 & 0.9672 \\
\hline 5 & 0.9236 & 0.8511 & 0.8493 & 0.8489 & 0.8489 & 1.6948 & 0.9774 & 0.9770 & 0.9703 & 0.9703 \\
\hline 10 & 0.9462 & 0.8364 & 0.8354 & 0.8352 & 0.8352 & 1.7835 & 0.9766 & 0.9764 & 0.9723 & 0.9723 \\
\hline 20 & 0.9622 & 0.8259 & 0.8253 & 0.8252 & 0.8252 & 1.8473 & 0.9763 & 0.9762 & 0.9738 & 0.9738 \\
\hline 50 & 0.9762 & 0.8165 & 0.8163 & 0.8162 & 0.8162 & 1.9040 & 0.9762 & 0.9761 & 0.9750 & 0.9750 \\
\hline 100 & 0.9832 & 0.8118 & 0.8117 & 0.8117 & 0.8117 & 1.9324 & 0.9762 & 0.9761 & 0.9755 & 0.9755 \\
\hline 200 & 0.9881 & 0.8085 & 0.8084 & 0.8084 & 0.8084 & 1.9524 & 0.9762 & 0.9762 & 0.9759 & 0.9759 \\
\hline 500 & 0.9925 & 0.8056 & 0.8055 & 0.8055 & 0.8055 & 1.9700 & 0.9762 & 0.9762 & 0.9761 & 0.9761 \\
\hline
\end{tabular}

\section{Lemma 6 There is the inequality}

$$
\frac{U B}{L B} \leq \exp \left\{\frac{1}{12 s} \sum_{l=1}^{\infty} \frac{1}{l^{2}} \frac{1}{\sqrt{2 \pi}} \int_{\alpha \sqrt{l}}^{\infty} e^{-\frac{1}{2} x^{2}} \mathrm{~d} x\right\}
$$


Proof Follows from (108)-(109) and $y^{\prime}(x) \leq 1$ (see Lemma 15).

We next show that the right-hand side of (112) is a decreasing function of $\lambda$ when $\beta>0$ is kept fixed. Indeed, this is a direct consequence of the following lemma.

Lemma $7 \alpha=(-2 s(1-\rho+\ln \rho))^{1 / 2}$ increases as a function of $\lambda$ when $\beta>0$ is kept fixed. In fact, $\alpha$ increases from 0 to $\beta$ as $\lambda$ increases from 0 to $\infty$.

Proof Letting $v=\frac{1}{2} \alpha^{2}$ we have

$$
\begin{aligned}
v & =-s(1-\rho+\ln \rho)=-(\lambda+\beta \sqrt{\lambda})\left(1-\frac{\lambda}{\lambda+\beta \sqrt{\lambda}}+\ln \frac{\lambda}{\lambda+\beta \sqrt{\lambda}}\right) \\
& =-\beta \sqrt{\lambda}-(\lambda+\beta \sqrt{\lambda}) \ln \frac{\lambda}{\lambda+\beta \sqrt{\lambda}}=-x\left(\beta+(x+\beta) \ln \left(\frac{x}{x+\beta}\right)\right),
\end{aligned}
$$

where we have set $x=\sqrt{\lambda}$. Now

$$
\begin{aligned}
\frac{\mathrm{d} v}{\mathrm{~d} x} & =-\beta-(x+\beta) \ln \left(\frac{x}{x+\beta}\right)-x\left(\ln \left(\frac{x}{x+\beta}\right)+(x+\beta) \cdot \frac{x+\beta}{x} \cdot \frac{\beta}{(x+\beta)^{2}}\right) \\
& =-2 \beta-(2 x+\beta) \ln \left(\frac{x}{x+\beta}\right)=2 x\left(-t+\left(1+\frac{1}{2} t\right) \ln (1+t)\right),
\end{aligned}
$$

where we have set $t=\beta / x>0$. Since it holds that

$$
\ln (1+t) \geq \frac{t}{1+\frac{1}{2} t}, \quad t \geq 0,
$$

(equality at $t=0$ ) and thus

$$
(\ln (1+t))^{\prime}=\frac{1}{1+t} \geq \frac{1}{\left(1+\frac{1}{2} t\right)^{2}}=\left(\frac{t}{1+\frac{1}{2} t}\right)^{\prime}, \quad t \geq 0,
$$

the proof is complete.

Lemma 8 The following inequalities hold:

(i) For $\alpha>0$,

$$
\frac{U B}{L B} \leq \exp \left\{\frac{\pi^{2}}{144 s}\right\}
$$

(ii) For $0<\alpha<2 \sqrt{\pi}$,

$$
\frac{U B}{L B} \leq \exp \left\{\frac{1}{12 s}\left[\frac{\pi^{2}}{12}+\frac{1}{2} \alpha^{2}-\frac{1}{\sqrt{2 \pi}} \sum_{r=0}^{\infty} \frac{\zeta(3 / 2-r)(-1 / 2)^{r}}{r !(2 r+1)} \alpha^{2 r+1}\right]\right\}
$$


Proof (i) Follows from (112) and observing that

$$
\sum_{l=1}^{\infty} \frac{1}{l^{2}} \frac{1}{\sqrt{2 \pi}} \int_{\alpha \sqrt{l}}^{\infty} e^{-\frac{1}{2} x^{2}} \mathrm{~d} x \leq \frac{1}{2} \sum_{l=1}^{\infty} \frac{1}{l^{2}}=\frac{1}{2} \zeta(2)=\frac{1}{12} \pi^{2} .
$$

(ii) Follows from rewriting the right-hand side of (112) in terms of Lerch's transcendent and applying the Bateman series (63).

Lemma 9 There are the inequalities

$$
\begin{aligned}
& L B \geq \exp \left\{-\sum_{l=1}^{\infty} \frac{1}{l} \frac{1}{\sqrt{2 \pi}} \int_{\alpha \sqrt{l}}^{\infty} e^{-\frac{1}{2} x^{2}} \mathrm{~d} x\right\} \\
& L B \leq \exp \left\{-\sum_{l=1}^{\infty} \frac{1}{l} \frac{1}{\sqrt{2 \pi}} \int_{\alpha \sqrt{l}}^{\infty} e^{-\frac{1}{2} x^{2}} \mathrm{~d} x+\frac{2}{3 \sqrt{2 \pi s}} \sum_{l=1}^{\infty} \frac{1}{l^{3 / 2}} e^{-\frac{1}{2} \alpha^{2} l}\right\} .
\end{aligned}
$$

Proof Follows from (108) and $1-\frac{2}{3} x \leq y^{\prime}(x) \leq 1$ (see Lemma 15).

Note that the right-hand side of (120) equals $\mathbb{P}\left(M_{\alpha}=0\right)$, i.e., the probability that the maximum of a Gaussian random walk with drift $-\alpha$ (instead of $-\beta$ ) equals zero.

\section{Lemma 10}

$$
\begin{aligned}
U B \leq & \exp \left\{-\sum_{l=1}^{\infty} \frac{1}{l} \frac{1}{\sqrt{2 \pi}} \int_{\alpha \sqrt{l}}^{\infty} e^{-\frac{1}{2} x^{2}} \mathrm{~d} x+\frac{1}{12} \sum_{l=1}^{\infty} \frac{1}{l^{2}} \frac{1}{\sqrt{2 \pi}} \int_{\alpha \sqrt{l}}^{\infty} e^{-\frac{1}{2} x^{2}} \mathrm{~d} x\right. \\
& \left.+\frac{2}{3 \sqrt{2 \pi s}} \sum_{l=1}^{\infty} \frac{1}{l^{3 / 2}} e^{-\frac{1}{2} \alpha^{2} l}-\frac{1}{18 s \sqrt{2 \pi s}} \sum_{l=1}^{\infty} \frac{1}{l^{3 / 2}} e^{-\frac{1}{2} \alpha^{2} l}\right\} .
\end{aligned}
$$

Proof Follows from (109) and $1-\frac{2}{3} x \leq y^{\prime}(x)$ (see Lemma 15).

The right-hand side of (122) can be written as

$$
\begin{aligned}
& \exp \left\{-\sum_{l=1}^{\infty} l^{-1} P(-\alpha \sqrt{l})+\frac{1}{12} \sum_{l=1}^{\infty} l^{-2} P(-\alpha \sqrt{l})\right. \\
& \left.+e^{-\frac{1}{2} \alpha^{2}} \Phi\left(e^{-\frac{1}{2} \alpha^{2}}, \frac{3}{2}, 1\right)\left(\frac{12 s-1}{18 s \sqrt{2 \pi s}}\right)\right\} .
\end{aligned}
$$

Using Lemma 4 for $k=-1,-2$, and (63), we find that (123) satisfies, for $\alpha<2 \sqrt{\pi}$,

$$
\begin{aligned}
& \sqrt{2} \alpha \exp \left\{\frac{\pi^{2}}{144}+\zeta\left(\frac{3}{2}\right)\left(\frac{12 s-1}{18 s \sqrt{2 \pi s}}\right)\right. \\
& \left.\quad+\alpha\left[\frac{\zeta\left(\frac{1}{2}\right)}{\sqrt{2 \pi}}-\frac{\zeta\left(\frac{3}{2}\right)}{12 \sqrt{2 \pi}}-\sqrt{2 \pi}\left(\frac{12 s-1}{18 s \sqrt{2 \pi s}}\right)\right]+\mathcal{O}\left(\alpha^{2}\right)\right\},
\end{aligned}
$$


which yields a sharp approximation for (123) for small values of $\alpha$.

Considering the leading component in the exponents of (121) and (122), it makes sense to use the approximation

$$
\begin{aligned}
\mathbb{P}\left(Q_{\lambda}=0\right) & \approx \exp \left\{-\sum_{l=1}^{\infty} \frac{1}{l} \frac{1}{\sqrt{2 \pi}} \int_{\alpha \sqrt{l}}^{\infty} e^{-\frac{1}{2} x^{2}} \mathrm{~d} x+\frac{2}{3 \sqrt{2 \pi s}} \sum_{l=1}^{\infty} \frac{1}{l^{3 / 2}} e^{-\frac{1}{2} \alpha^{2} l}\right\} \\
& =\mathbb{P}\left(M_{\alpha}=0\right) \cdot \exp \left\{\frac{2}{3 \sqrt{2 \pi s}} \sum_{l=1}^{\infty} \frac{1}{l 3 / 2} e^{-\frac{1}{2} \alpha^{2} l}\right\} \\
& =\mathbb{P}\left(M_{\alpha}=0\right) \cdot \exp \left\{\frac{2}{3 \sqrt{2 \pi s}} e^{-\frac{1}{2} \alpha^{2}} \Phi\left(e^{-\frac{1}{2} \alpha^{2}}, \frac{3}{2}, 1\right)\right\} .
\end{aligned}
$$

We note that the approximations (107) and (125) are strongly related. In fact, approximation (125) follows from (107) by approximating $y^{\prime}(x)$ by $1-\frac{2}{3} x$.

From (1), (63) and (125) we then get, for $\beta<2 \sqrt{\pi}$ (and hence $\alpha<2 \sqrt{\pi}$ ),

$$
\begin{aligned}
\frac{\mathbb{P}\left(Q_{\lambda}=0\right)}{\mathbb{P}\left(M_{\beta}=0\right)}= & \frac{\mathbb{P}\left(M_{\alpha}=0\right)}{\mathbb{P}\left(M_{\beta}=0\right)} \cdot \exp \left\{\frac{2}{3 \sqrt{2 \pi s}} e^{-\frac{1}{2} \alpha^{2}} \Phi\left(e^{-\frac{1}{2} \alpha^{2}}, \frac{3}{2}, 1\right)\right\} \\
\approx & \frac{\alpha}{\beta} \cdot \exp \left\{\frac{1}{\sqrt{2 \pi}} \sum_{r=0}^{\infty} \frac{\zeta\left(\frac{1}{2}-r\right)\left(-\frac{1}{2}\right)^{r}}{r !(2 r+1)}\left(\alpha^{2 r+1}-\beta^{2 r+1}\right)\right\} \\
& \times \exp \left\{\frac{2}{3 \sqrt{2 \pi s}}\left(-\sqrt{2 \pi} \alpha+\sum_{r=0}^{\infty} \frac{\zeta\left(\frac{3}{2}-r\right)\left(-\frac{1}{2}\right)^{r}}{r !} \alpha^{2 r}\right)\right\}
\end{aligned}
$$

For small values of $\alpha$ we then propose the approximation

$$
\frac{\mathbb{P}\left(Q_{\lambda}=0\right)}{\mathbb{P}\left(M_{\beta}=0\right)} \approx \frac{\alpha}{\beta} \cdot \exp \left\{\frac{\zeta\left(\frac{1}{2}\right)}{\sqrt{2 \pi}}(\alpha-\beta)+\frac{2}{3}\left(\frac{\zeta\left(\frac{3}{2}\right)}{\sqrt{2 \pi}}-\alpha\right) \frac{1}{\sqrt{s}}\right\}
$$

\subsection{Numerical experiments}

We now evaluate the approximations and bounds derived in this section. True values of $\mathbb{P}\left(Q_{\lambda}=0\right)$ are computed from (17). Some numerical results are displayed in Tables 4 and 5. In Table 4 , the values of $\mathbb{P}\left(M_{\beta}=0\right)$ are given in parentheses.

Both the lower bound $\mathbb{P}\left(M_{\alpha}=0\right)$ and the upper bound (122) may seem conservative approximations, but the quality of approximation (125) is striking for small and moderate values of $\beta$. For large values, it seems that the limiting value is reached quite rapidly. In particular, for the case $\beta=2$, the Halfin-Whitt limit $\mathbb{P}\left(M_{\beta}=0\right)$ is reached almost immediately, which suggests to use $\mathbb{P}\left(M_{\beta}=0\right)$ as an approximation for $\mathbb{P}\left(Q_{\lambda}=0\right)$. Indeed, as such, the Halfin-Whitt limit outperforms all other approximations, including the series expansions in Table 3. For all other values of $\beta$ (especially small values), all alternative approximations of the emptiness probability are closer to the true values than the Halfin-Whitt limit. 
Table 4 Bounds, approximations and true values of $\mathbb{P}\left(Q_{\lambda}=0\right)$

\begin{tabular}{|c|c|c|c|c|c|c|c|c|c|c|}
\hline \multirow[t]{2}{*}{$s$} & \multirow[t]{2}{*}{$\alpha$} & \multicolumn{4}{|c|}{$\beta=0.01(0.0141)$} & \multirow[t]{2}{*}{$\alpha$} & \multicolumn{4}{|c|}{$\beta=0.1(0.1334)$} \\
\hline & & $(120)$ & True & (125) & (122) & & $(120)$ & True & (125) & (122) \\
\hline 1 & 0.0100 & 0.0140 & 0.0268 & 0.0279 & 0.0282 & 0.0983 & 0.1313 & 0.2351 & 0.2468 & 0.2488 \\
\hline 2 & 0.0100 & 0.0140 & 0.0225 & 0.0228 & 0.0240 & 0.0988 & 0.1319 & 0.2022 & 0.2061 & 0.2149 \\
\hline 5 & 0.0100 & 0.0140 & 0.0190 & 0.01910 & 0.0203 & 0.0993 & 0.1325 & 0.1747 & 0.1756 & 0.1857 \\
\hline 10 & 0.0100 & 0.0141 & 0.0174 & 0.0175 & 0.0187 & 0.0995 & 0.1328 & 0.1617 & 0.1620 & 0.1718 \\
\hline 20 & 0.0100 & 0.0141 & 0.0164 & 0.0164 & 0.0175 & 0.0996 & 0.1329 & 0.1529 & 0.1531 & 0.1625 \\
\hline 50 & 0.0100 & 0.0141 & 0.0155 & 0.0155 & 0.0166 & 0.0998 & 1331 & 0.1455 & 0.1455 & 0.1546 \\
\hline 100 & 0.0100 & 0.0141 & 0.0151 & 0.0151 & 0.0161 & 0.0998 & 0.1332 & 0.1419 & 0.1419 & 0.1507 \\
\hline 200 & 0.0100 & 0.0141 & 0.0148 & 0.0148 & 0.0158 & 0.0999 & 0.1333 & 0.1393 & 0.1393 & 0.148 \\
\hline 500 & 0.0100 & 0.0141 & 0.0145 & 0.0145 & 0.0155 & 0.0999 & 0.1333 & 0.1371 & 0.1371 & 0.1457 \\
\hline & \multirow[t]{2}{*}{$\alpha$} & \multicolumn{4}{|c|}{$\beta=0.2(0.2518)$} & $\alpha$ & \multicolumn{4}{|c|}{$\beta=0.5(0.5293)$} \\
\hline & & (120) & True & (125) & (122) & & (120) & True & (125) & (122) \\
\hline 1 & 0.1932 & 0.2442 & 0.4105 & 0.4332 & 0.4356 & 0.4573 & 0.4961 & 0.7182 & 0.7628 & 0.7635 \\
\hline 2 & 0.1952 & 0.2464 & 0.3613 & 0.3693 & 0.3829 & 0.4699 & 0.5061 & 0.6656 & 0.6831 & 0.6994 \\
\hline 5 & 0.1970 & 0.2484 & 0.3185 & 0.3207 & 0.3367 & 0.4811 & 0.5148 & 0.6156 & 0.6208 & 0.6413 \\
\hline 10 & 0.1979 & 0.2494 & 0.2979 & 0.2987 & 0.3145 & 0.4867 & 0.5192 & 0.5899 & 0.5921 & 0.6127 \\
\hline 20 & 0.1985 & 0.2501 & 0.2838 & 0.2841 & 0.2994 & 0.4906 & & 0.5719 & 0.5728 & 0.5930 \\
\hline 50 & 0.1991 & 0.2507 & 0.2716 & & 0.2865 & 0.4941 & & 0.5560 & & 0.5760 \\
\hline 100 & 0.1993 & 0.2510 & 0.2657 & 0.2657 & 0.2801 & 0.4958 & 0.5262 & 0.5481 & 0.5483 & 0.5676 \\
\hline 200 & 0.1995 & 0.2512 & 0.2615 & 0.2616 & 0.2757 & 0.4970 & 0.5271 & 0.5426 & 0.5426 & 0.5618 \\
\hline 500 & 0.1997 & 0.2514 & 0.2579 & & & 0.4981 & & 0.5377 & 0.5377 & 0.5566 \\
\hline & \multirow[t]{2}{*}{$\alpha$} & \multicolumn{4}{|c|}{$\beta=1(0.8005)$} & $\alpha$ & \multicolumn{4}{|c|}{$\beta=2(0.9762)$} \\
\hline & & (120) & True & (125) & (122) & & (120) & True & (125) & (122) \\
\hline 1 & 0.8299 & 0.7294 & 0.9055 & 0.9574 & 0.9554 & 1.3670 & 0.9019 & 0.9835 & 1.0202 & 1.0177 \\
\hline 2 & 0.8790 & 0.7518 & 0.8787 & 0.9002 & 0.9106 & 1.5296 & 0.9302 & 0.9799 & 0.9938 & 0.9966 \\
\hline 5 & 0.9236 & 0.7709 & 0.8511 & 0.8581 & 0.8718 & 1.6948 & 0.9514 & 0.9774 & 0.9814 & 0.9847 \\
\hline 10 & 0.9462 & 0.7800 & 0.8364 & 0.8395 & 0.8533 & 1.7835 & 0.9603 & 0.9766 & 0.9782 & 0.9812 \\
\hline 20 & 0.9622 & 0.7863 & 0.8259 & 0.8273 & 0.8408 & 1.8473 & 0.9657 & 0.9763 & 0.9770 & 0.9797 \\
\hline 50 & 0.9762 & 0.7917 & 0.8165 & 0.8170 & 0.8302 & 1.9040 & 0.9700 & 0.9762 & 0.9764 & 0.9788 \\
\hline 100 & 0.9832 & 0.7943 & 0.8118 & 0.8120 & 0.8250 & 1.9324 & 0.9720 & 0.9762 & 0.9763 & 0.9785 \\
\hline 200 & 0.9881 & 0.7962 & 0.8085 & 0.8086 & 0.8214 & 1.9524 & 0.9733 & 0.9762 & 0.9762 & 0.9784 \\
\hline 500 & 0.9925 & 0.7978 & 0.8056 & 0.8056 & 0.8183 & 1.9700 & 0.9745 & 0.9762 & 0.9762 & 0.9783 \\
\hline
\end{tabular}

\section{Conclusions and outlook}

The approach in this paper consists of three major steps:

1. Quasi-Gaussian form. Section 3 is the bridge that permits us to pass from the general form of Spitzer's formula (16) to the more convenient quasi-Gaussian 
Table 5 Bounds, approximations and true values of $\mathbb{P}\left(Q_{\lambda}=0\right) / \mathbb{P}\left(M_{\beta}=0\right)$

\begin{tabular}{|c|c|c|c|c|c|c|c|c|c|c|}
\hline \multirow[t]{2}{*}{$s$} & \multirow[t]{2}{*}{ True } & \multicolumn{4}{|c|}{$\beta=0.01$} & \multirow[t]{2}{*}{ True } & \multicolumn{4}{|l|}{$\beta=0.1$} \\
\hline & & (126) & $\mathrm{lb}(18)$ & $\mathrm{ub}(18)$ & (23) & & (126) & $\mathrm{lb}(18)$ & $\mathrm{ub}(18)$ & (23) \\
\hline 1 & 1.9047 & 1.9870 & 0.1224 & 8.1692 & 1.1868 & 1.7622 & 1.8500 & 0.1111 & 8.9980 & 1.1608 \\
\hline 2 & 1.5993 & 1.6251 & 0.2270 & 4.4062 & 1.1285 & 1.5154 & 1.5447 & 0.2163 & 4.6226 & 1.1095 \\
\hline 5 & 1.3537 & 1.3595 & 0.3919 & 2.5516 & 1.0794 & 1.3092 & 1.3164 & 0.3845 & 2.6007 & 1.0670 \\
\hline 10 & 1.2406 & 1.2426 & 0.5159 & 1.9385 & 1.0554 & 1.2119 & 1.2145 & 0.5110 & 1.9570 & 1.0466 \\
\hline 20 & 1.1653 & 1.1661 & 0.6264 & 1.5965 & 1.0389 & 1.1463 & 1.1473 & 0.6234 & 1.6041 & 1.0326 \\
\hline 50 & 1.1018 & 1.1021 & 0.7440 & 1.3442 & 1.0244 & 1.0905 & 1.0908 & 0.7426 & 1.3467 & 1.0204 \\
\hline 100 & 1.0710 & 1.0712 & 0.8113 & 1.2326 & 1.0172 & 1.0633 & 1.0634 & 0.8106 & 1.2337 & 1.0144 \\
\hline 200 & 1.0497 & 1.0499 & 0.8626 & 1.1593 & 1.0121 & 1.0444 & 1.0444 & 0.8622 & 1.1599 & 1.0101 \\
\hline 500 & 1.0312 & 1.0313 & 0.9108 & 1.0980 & 1.0077 & 1.0279 & 1.0279 & 0.9106 & 1.0982 & 1.0064 \\
\hline \multirow[t]{2}{*}{$S$} & \multirow[t]{2}{*}{ True } & \multicolumn{4}{|l|}{$\beta=0.2$} & \multirow[t]{2}{*}{ True } & \multicolumn{4}{|l|}{$\beta=0.5$} \\
\hline & & (126) & $\mathrm{lb}(18)$ & $\mathrm{ub}(18)$ & (23) & & (126) & $\mathrm{lb}(18)$ & ub(18) & (23) \\
\hline 1 & 1.6307 & 1.7207 & 0.0993 & 10.0675 & 1.1328 & 1.3568 & 1.4411 & 0.0688 & 14.537 & 1.0565 \\
\hline 2 & 1.4351 & 1.4669 & 0.2047 & 4.8840 & 1.0894 & 1.2575 & 1.2905 & 0.1717 & 5.8237 & 1.0368 \\
\hline 5 & 1.2652 & 1.2738 & 0.3763 & 2.6574 & 1.0542 & 1.1629 & 1.1729 & 0.3517 & 2.8433 & 1.0217 \\
\hline 10 & 1.1831 & 1.1865 & 0.5055 & 1.9781 & 1.0375 & 1.1145 & 1.1187 & 0.4891 & 2.0446 & 1.0148 \\
\hline 20 & 1.1271 & 1.1284 & 0.6201 & 1.6127 & 1.0261 & 1.0803 & 1.0822 & 0.6101 & 1.6391 & 1.0102 \\
\hline 50 & 1.0789 & 1.0794 & 0.7410 & 1.3495 & 1.0163 & 1.0504 & 1.0511 & 0.7362 & 1.3582 & 1.0063 \\
\hline 100 & 1.0553 & 1.0555 & 0.8097 & 1.2350 & 1.0115 & 1.0355 & 1.0358 & 0.8071 & 1.2390 & 1.0044 \\
\hline 200 & 1.0388 & 1.0389 & 0.8617 & 1.1605 & 1.0081 & 1.0250 & 1.0251 & 0.8604 & 1.1623 & 1.0031 \\
\hline 500 & 1.0244 & 1.0244 & 0.9104 & 1.0984 & 1.0051 & 1.0158 & 1.0158 & 0.9098 & 1.0991 & 1.0019 \\
\hline \multirow[t]{2}{*}{$s$} & \multirow[t]{2}{*}{ True } & \multicolumn{4}{|l|}{$\beta=1$} & \multirow[t]{2}{*}{ True } & \multicolumn{4}{|l|}{$\beta=2$} \\
\hline & & (126) & $\mathrm{lb}(18)$ & $\mathrm{ub}(18)$ & (23) & & (126) & $\mathrm{lb}(18)$ & $\mathrm{ub}(18)$ & (23) \\
\hline 1 & 1.1311 & 1.1960 & 0.0340 & 29.4157 & 0.9645 & 1.0074 & 1.0450 & 0.0064 & 155.3166 & 0.9292 \\
\hline 2 & 1.0976 & 1.1245 & 0.1237 & 8.0841 & 0.9779 & 1.0037 & 1.0179 & 0.0576 & 17.3720 & 0.9593 \\
\hline 5 & 1.0632 & 1.0719 & 0.3114 & 3.2114 & 0.9876 & 1.0011 & 1.0052 & 0.2365 & 4.2284 & 0.9792 \\
\hline 10 & 1.0448 & 1.0487 & 0.4614 & 2.1675 & 0.9918 & 1.0003 & 1.0020 & 0.4057 & 2.4649 & 0.9870 \\
\hline 20 & 1.0316 & 1.0334 & 0.5931 & 1.6862 & 0.9944 & 1.0000 & 1.0007 & 0.5580 & 1.7920 & 0.9915 \\
\hline 50 & 1.0199 & 1.0206 & 0.7282 & 1.3733 & 0.9966 & 1.0000 & 1.0001 & 0.7116 & 1.4054 & 0.9951 \\
\hline 100 & 1.0141 & 1.0144 & 0.8028 & 1.2457 & 0.9977 & 1.0000 & 1.0000 & 0.7938 & 1.2598 & 0.9966 \\
\hline 200 & 1.0099 & 1.0101 & 0.8580 & 1.1654 & 0.9984 & 1.0000 & 1.0000 & 0.8533 & 1.1719 & 0.9977 \\
\hline 500 & 1.0063 & 1.0063 & 0.9088 & 1.1003 & 0.9990 & 1.0000 & 1.0000 & 0.9069 & 1.1027 & 0.9986 \\
\hline
\end{tabular}

form (24). The key facilitator is, see (42),

$$
\mathbb{P}\left(A_{\lambda} \leq s\right)=\frac{p(s) \sqrt{s}}{\sqrt{2 \pi}} \int_{-\infty}^{\alpha / \sqrt{s}} e^{-\frac{1}{2} s x^{2}} y^{\prime}(x) \mathrm{d} x,
$$

where, as before, $A_{\lambda}$ denotes a Poisson random variable with mean $\lambda$. 
2. Analysis of the function $y$. The appendix contains a detailed study of the function $y\left(\right.$ and $\left.y^{\prime}\right)$. It is shown that there is a strong connection with the Gamma function, the reciprocal Gamma function and the Lambert $W$ function. For this paper, the most important result is the power series for $y$, see (149), which is shown to have rapidly decaying coefficients. The latter property makes the quasi-Gaussian form (128) extremely effective, because only the first few terms of $y^{\prime}$ have to be taken into account.

3. Principal series expansions. Starting from the quasi-Gaussian form, we derive in Section 4 principal series expansions for $\mathbb{P}\left(Q_{\lambda}=0\right)$. The expansions comprise elementary functions and are used to derive asymptotic expressions and bounds for $\mathbb{P}\left(Q_{\lambda}=0\right)$. The power series for $y$ leads to even more explicit expansions.

Steps 1 and 2 are very case-specific, requiring properties of the Poisson distribution and the associated function $y^{\prime}$. Step 3 is far more general. The expansions presented hold for a large class of functions $z$, of which $z=y^{\prime}$ (Poisson case) and $z \equiv 1$ (Gaussian random walk) are just special cases. We now briefly indicate some ideas that enable the extension of the first two steps. A more general version of our model is

$$
Q_{\lambda} \stackrel{d}{=}\left(Q_{\lambda}+A_{\lambda}^{*}-s\right)^{+} .
$$

If we can write the random variable $A_{\lambda}^{*}$ as the sum of $s$ i.i.d. random variables, which each converge weakly to a random variable with unit mean as $\lambda \rightarrow \infty$, then under some additional assumptions (for example a Lindeberg-type condition) one has still weak convergence of $\left(A_{\lambda}^{*}-s\right) / \sqrt{s}$ to a Gaussian random variable with negative mean. To write the associated performance measure, such as the emptiness probability, in a convenient form, our method of attack would still work if the cumulative distribution function $\mathbb{P}\left(A_{\lambda}^{*} \leq s\right)$ is of type (128) with $y^{\prime}(x)$ replaced by some function that depends on $A_{\lambda}^{*}$. Temme [30] describes a large class of distributions that can be brought in this shape.

Acknowledgements We thank M. Vlasiou for pointing out a connection to the Lambert W function. JvL was supported by the Netherlands Organisation for Scientific Research (NWO).

Open Access This article is distributed under the terms of the Creative Commons Attribution Noncommercial License which permits any noncommercial use, distribution, and reproduction in any medium, provided the original author(s) and source are credited.

\section{Appendix: Analysis of the function $y$}

We shall present an analysis to obtain some results on the function $y(x)$ that appeared in Sect. 2.2, especially in (24). As before, $y(x)$ is, for $|x|$ sufficiently small, the solution $y$ of the equation

$$
f(y):=-y-\ln (1-y)=\frac{1}{2} x^{2}
$$

that is real and positive when $x$ is real, positive and sufficiently small. We note that for $|y|<1$,

$$
f(y)=\frac{1}{2} y^{2}+\frac{1}{3} y^{3}+\frac{1}{4} y^{4}+\cdots,
$$


whence we can write (129) for small $x$ and $y$ as

$$
y\left(1+\frac{2}{3} y+\frac{1}{2} y^{2}+\cdots\right)^{1 / 2}=x
$$

with the principal value of the square root. Hence,

$$
y(x)=x-\frac{1}{3} x^{2}+\mathcal{O}\left(x^{3}\right) .
$$

From (129) we furthermore see that $y(x)$ increases from 0 to $\infty$ as $x$ increases from 0 to 1 , and that $y(x)$ increases from $-\infty$ to 0 as $x$ increases from $-\infty$ to 0 .

The function $y(x)$ occurs in connection with the asymptotic expansions of $\Gamma(t+1)$ and $1 / \Gamma(t)$ as $t \rightarrow \infty$. Thus one substitutes in the integral representation

$$
\Gamma(t+1)=\int_{0}^{\infty} e^{-u} u^{t} \mathrm{~d} u
$$

subsequently $u=t(1+v), v=-y$ and $y=y(x)$ to obtain

$$
\begin{aligned}
\Gamma(t+1) & =e^{-t} t^{t+1} \int_{-1}^{\infty} e^{t(-v+\ln (1+v))} \mathrm{d} v \\
& =e^{-t} t^{t+1} \int_{-\infty}^{1} e^{-t f(y)} \mathrm{d} y=e^{-t} t^{t+1} \int_{-\infty}^{\infty} e^{-\frac{1}{2} t x^{2}} y^{\prime}(x) \mathrm{d} x .
\end{aligned}
$$

We refer to [8], Sect. 4.5 on pp. 69-71, and to [23], Chap. 3, Sect. 8 on pp. 85-86. By (131), we have that $y(x)$ has a power series

$$
y(x)=\sum_{n=1}^{\infty} a_{n} x^{n},
$$

converging for $|x|$ sufficiently small. Then by Watson's lemma, see e.g. [23], pp. 112116 , the asymptotics of $\Gamma(t+1)$ follows from (134) as

$$
\begin{aligned}
\Gamma(t+1) & \sim e^{-t} t^{t+1} \sum_{n=1}^{\infty} n a_{n} \int_{-\infty}^{\infty} x^{n-1} e^{-\frac{1}{2} t x^{2}} \mathrm{~d} x \\
& =e^{-t} t^{t+1} \sqrt{2 \pi} \sum_{k=0}^{\infty} \frac{(2 k+1)(2 k-1) \cdots 1}{t^{k}} a_{2 k+1},
\end{aligned}
$$

as $t \rightarrow \infty$. Note that the terms with odd $n$ in the first series in (136) vanish. In a similar fashion the asymptotics of $1 / \Gamma(t)$ can be obtained from the Hankel representation

$$
\frac{1}{\Gamma(t)}=\frac{i}{2 \pi} \int_{\mathcal{C}}(-u)^{-t} e^{-u} \mathrm{~d} u,
$$

where $\mathcal{C}$ is a contour that starts at $\infty+i 0$, encircles the origin in positive sense and ends at $\infty-i 0$. This is carried out in [31], Sect. 3.6.3 on pp. 69-70. Thus by 
substitutions as in (134) we now get

$$
\frac{1}{\Gamma(t)}=\frac{i}{2 \pi} \frac{1}{e^{-t} t^{t-1}} \int_{1+\mathcal{C}} e^{t f(y)} \mathrm{d} y,
$$

where it is observed that $\operatorname{Re} f(y) \rightarrow-\infty$ as $y \rightarrow \infty \pm i 0$. Now the integration path $1+\mathcal{C}$ is deformed so as to pass through the saddle point $y=0$ where the real axis is crossed perpendicularly from the upper half plane into the lower half plane. Around the saddle point we make the substitution $f(y)=-\frac{1}{2} x^{2}=\frac{1}{2}(i x)^{2}$ where $x \in \mathbb{R}$ decreases from $+\delta$ to $-\delta$, with $\delta>0$ sufficiently small, and we get, as $t \rightarrow \infty$,

$$
\begin{aligned}
\frac{1}{\Gamma(t)} & \sim \frac{1}{2 \pi} \frac{1}{e^{-t} t^{t-1}} \int_{-\delta}^{\delta} e^{-\frac{1}{2} t x^{2}} y^{\prime}(i x) \mathrm{d} x \\
& \sim \frac{1}{2 \pi} \frac{1}{e^{-t} t^{t-1}} \sum_{n=1}^{\infty} n a_{n} i^{n-1} \int_{-\delta}^{\delta} x^{n-1} e^{-\frac{1}{2} t x^{2}} \mathrm{~d} x \\
& \sim \frac{1}{e^{-t} t^{t-1 / 2} \sqrt{2 \pi}} \sum_{k=0}^{\infty}(-1)^{k} \frac{(2 k+1)(2 k-1) \cdots 1}{t^{k}} a_{2 k+1} .
\end{aligned}
$$

In particular, we have that the asymptotics of the $p(n)$ of (27) is given by

$$
p(n)=\frac{n^{n-1} e^{-n} \sqrt{2 \pi n}}{\Gamma(n)} \sim \sum_{k=0}^{\infty}(-1)^{k} \frac{(2 k+1)(2 k-1) \cdots 1}{n^{k}} a_{2 k+1}, \quad n \rightarrow \infty
$$

so that the $p_{k}$ 's in (27) are given as

$$
p_{k}=(-1)^{k}(2 k+1)(2 k-1) \cdots 1 \cdot a_{2 k+1}, \quad k=0,1, \ldots
$$

We shall now present and establish some results for the coefficients $a_{n}$ of the power series $\sum_{n=1}^{\infty} a_{n} x^{n}$ of $y(x)$. In [34], p. 16, there is given the result

$$
\frac{1}{\Gamma(t+1)} \sim \frac{e^{t}}{\pi t^{t+1 / 2}} \sum_{k=0}^{\infty} \frac{c_{k} \Gamma(k+3 / 2)}{t^{k}}, \quad t \rightarrow \infty
$$

with $c_{k}$ the coefficients of a function $\phi(\tau)$ that is regular inside the circle $|\tau|=2 \pi$. Apparently, since $t \Gamma(t)=\Gamma(t+1)$ and $\Gamma(k+3 / 2)=(k+1 / 2)(k-1 / 2) \cdots \frac{1}{2} \cdot \sqrt{\pi}$, we have that

$$
a_{2 k+1}=(-1)^{k} 2^{-k-1 / 2} c_{k}, \quad k=0,1, \ldots,
$$

so that $a_{2 k+1}$ decays like $\left(\frac{1}{4 \pi}+\epsilon\right)^{k}$ for any $\epsilon>0$. However, with the asymmetric integration ranges (unlike those in (134) and (139)) that occur in the integrals at the right-hand side of (24), the decay behaviour of the coefficients $a_{2 k}$ is equally important. We shall thus conduct an analysis for $y(x)$ that is somewhat similar to the analysis in [34], Sects. 7-9 on pp. 9-15, for the function $\phi(\tau)=\sum_{k=0}^{\infty} c_{k} \tau^{k}$. This analysis is greatly facilitated by the study of the properties of the mapping

$$
z \in \mathbb{C} \mapsto e^{1-z} z=w
$$


as given in Szegö [28]. Note that $\exp (f(y))=(1-y) e^{y}$ so that many of the observations of Szegö directly apply to our function $f$. The mapping given in (144) is clearly related to the Lambert $W$ function, which is the inverse of the mapping $W \in \mathbb{C} \mapsto W e^{W}$, see $[7,16]$. Explicitly, our $y(x)$ and Lambert's $W$ function are related according to

$$
y(x)=1+W\left(-e^{-\left(1+\frac{1}{2} x^{2}\right)}\right), \quad x>0 .
$$

It appears that Szegö's analysis of the mapping in (144) has largely escaped the attention of the Lambert $W$ community. In the proof of the lemma below, we heavily rely on this analysis and omit some of the details that are contained implicitly or explicitly in [28], Sect. 2.

Lemma 11 The functions $y(x)$ extends to an analytic function on all of $\mathbb{C}$ with the exception of two branch cuts from $2 \sqrt{\pi} \cdot e^{ \pm \frac{1}{4} \pi i}$ to $\infty \cdot e^{ \pm \frac{1}{4} \pi i}$.

Proof (sketch) Our approach is to take any angle $\theta$ with $-\pi \leq \theta \leq \pi$, and to see how far $y(x)$ admits analytic continuation when $x$ moves in the direction $e^{i \theta}$ from 0 . Clearly, from (132), $y(x)$ starts moving from 0 in the direction $e^{i \theta}$ when $x$ does so. We thus plot curves $C_{\theta}$ in the $y$-plane that start at the origin and leave from there under an angle $\theta \in[-\pi, \pi]$ with the positive real axis and on which $\arg [-y-$ $\ln (1-y)]=2 \theta$. Writing $y=1-z$ and $z=\eta e^{i \varphi}$ with $\eta>0$ and real $\varphi$, there should hold

$$
\eta \cos \varphi-1-\ln \eta+i(\eta \sin \varphi-\varphi)=r \cos 2 \theta+i r \sin 2 \theta
$$

with $r \geq 0$ for $y$ to lie on $C_{\theta}$. Consequently, we should have

$$
\frac{\eta \sin \varphi-\varphi}{\eta \cos \varphi-1-\ln \eta}=\tan 2 \theta .
$$

With reference to Fig. 2, we have that the cases with $\tan 2 \theta=0$ give rise to the curves with $\theta=0, \pm \pi / 2, \pm \pi$. The cases that $\tan 2 \theta=\infty$ give rise to the four curves with $\theta= \pm \pi / 4, \pm 3 \pi / 4$. We note that $f^{\prime}(y)=y /(1-y)$ so around any $y_{0} \neq 0,1$, the mapping $y \rightarrow f(y)$ is locally invertible as an analytic function. Furthermore, $|f(y)|$ increases along any of the curves $C_{\theta}$. Indeed, this is so, by (132), when $y$ is close to 0 , and $|f(y)|$ cannot have stationary points as $y \neq 0,1$ moves along $C_{\theta}$, for otherwise $\ln f(y)=\ln |f(y)|+2 i \theta+2 \pi i n$ ( $n$ some integer) would have a stationary point, contradicting $f^{\prime}(y) \neq 0$. Along the curves we define $\ln (1-y)$ as the integral of $-1 /(1-z)$ with $z$ moving along $C_{\theta}$ from 0 to $y$, and thus obtain a $\log (1-y)$ that is analytic around $C_{\theta}$. The curves with $\theta= \pm \pi / 4$ are particular since they re-enter the origin (where $f^{\prime}=0$ ), and therefore do not admit an analytic continuation of $y(x)$ beyond there. The values of $x$ at $y=0$ in these re-entrance cases are $i^{ \pm 1 / 2} \sqrt{2 \pi}$ for $\theta= \pm \pi / 4$, respectively, since $-y-\ln (1-y)$ has the respective values $\pm 2 \pi i$ at re-entry to zero along $C_{ \pm \pi / 4}$. The $C_{ \pm \pi / 4}$ constitute mirrored versions of Szegö's curve, see [28], Fig. 1 on p. 51, passed with negative orientation $(+\pi / 4)$ and positive orientation $(-\pi / 4)$, respectively. In the cases that $0 \leq|\theta|<\pi / 4$, we have that $C_{\theta}$ is bounded by the closed curves $C_{ \pm \pi / 4}$, and that $y \rightarrow 1$. The asymptotic behavior 
Fig. 2 Curves $C_{\theta}$ in the $y$-plane. $\varepsilon=0.1$

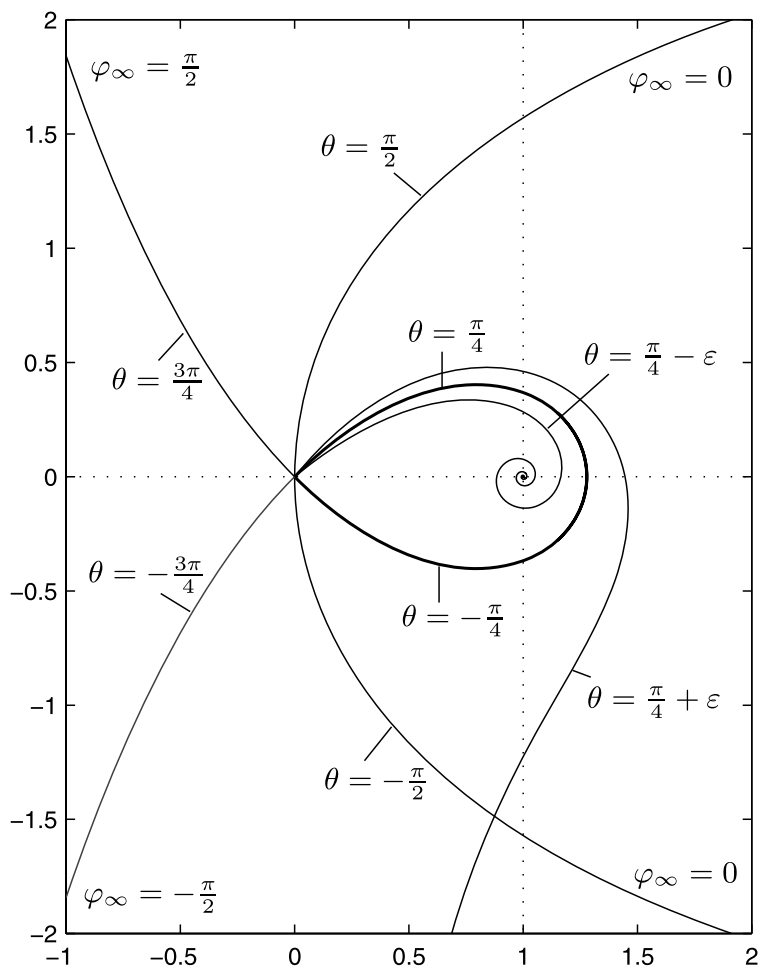

of $y$ is described in these cases in terms of $\eta, \varphi$ (see (146), (147)) by the spiral $\eta=$ $\exp (-1+\varphi / \tan 2 \theta)$ when $\varphi \cdot \operatorname{sgn}(\theta) \rightarrow \infty$ (observe that in these cases $\theta$ and $\varphi$ have opposite signs). Consequently, when $0<|\theta|<\pi / 4$, we have that $|x|=|2 f(y)|^{1 / 2} \rightarrow$ $\infty$ along the curve $C_{\theta}$. In the cases that $\frac{\pi}{4}<|\theta|<\pi$, we have that $C_{\theta}$ lies completely outside the region enclosed by $C_{ \pm \pi / 4}$, and along these curves we have that $|y| \rightarrow \infty$. Therefore $|\ln (1-y) / y| \rightarrow 0$ along these curves, and since we have required that $\arg [-y-\ln (1-y)]=2 \theta$, we have that

$$
\varphi_{\infty}:=\lim _{|y| \rightarrow \infty, y \in C_{\theta}} \arg (y)=2 \theta-\pi(\bmod [-\pi, \pi]) .
$$

Also, $|x|=|2 f(y)|^{1 / 2} \rightarrow \infty$ in these cases. We thus conclude that $y(x)$ can be continued analytically along all rays $x=r e^{i \theta}, r \geq 0$, when $\theta \in[-\pi, \pi]$, except for $\theta= \pm \pi / 4$. In the latter cases the analytic continuation can only be carried out until $r=2 \sqrt{\pi}$.

\section{Lemma 12 We have}

$$
y(x)=\sum_{n=1}^{\infty} a_{n} x^{n}, \quad|x|<2 \sqrt{\pi},
$$


with $a_{1}=1$ and the $a_{n}$ 's recursively defined as

$$
a_{k+2}=\frac{-1}{k+3}\left(a_{k+1}+\sum_{n=1}^{k}(n+1) a_{n+1} a_{k+2-n}\right), \quad k=0,1, \ldots
$$

Proof From Lemma 11 we have that $y(x)$ is analytic in the disk $|x|<2 \sqrt{\pi}$, with branch points at $x=2 \sqrt{\pi} \cdot i^{ \pm 1 / 2}$. This gives (149). From (129) we get by differentiation with respect to $x$ and some rewriting the equation

$$
y^{\prime}(x) y(x)=x-x y(x) .
$$

Using $a_{1}=1$, this can be written in terms of the $a_{n}$ 's as

$$
\sum_{n=0}^{k-1}(n+1) a_{n+1} a_{k-n}=-a_{k-1}, \quad k=2,3, \ldots,
$$

and this gives (150). Also see [23], Ex. 8.3 on p. 88 and [31], p. 70.

The first five coefficients $a_{n}$ are given by

$$
a_{1}=1, \quad a_{2}=-\frac{1}{3}, \quad a_{3}=\frac{1}{36}, \quad a_{4}=\frac{1}{270}, \quad a_{5}=\frac{1}{4320},
$$

and this rapid decay is further substantiated by the following result.

Lemma 13 There is the asymptotic form (rapid decay to 0 )

$$
a_{n}=\frac{\sin \frac{\pi}{4}(n-1)-\frac{2 \pi}{6 n-9} \cos \frac{\pi}{4}(n-1)}{\left(\frac{1}{2} n\right)^{3 / 2}(2 \sqrt{\pi})^{n}}(1+\mathcal{O}(1 / n)) .
$$

Proof The precise asymptotic behavior of the $a_{n}$ can be determined by Darboux's method, see Szegö [29], Sect. 8.4 on pp. 206-208. For this we examine $y\left(e^{ \pm \pi i / 4} v\right)$ with $0 \leq v<2 \sqrt{\pi}$ as the solution $y$ near 0 and on $C_{ \pm \pi / 4}$ of the equation

$$
2 \pi i-y-\ln (1-y)=2 \pi i+\frac{1}{2} y^{2}+\frac{1}{3} y^{3}+\cdots=\frac{1}{2} i v^{2} .
$$

Thus

$$
y\left(e^{\pi i / 4} v\right)=e^{-\pi i / 4} \sqrt{4 \pi-v^{2}}+\mathcal{O}(2 \sqrt{\pi}-v), \quad v \uparrow 2 \sqrt{\pi},
$$

and similarly,

$$
y\left(e^{-\pi i / 4} v\right)=e^{\pi i / 4} \sqrt{4 \pi-v^{2}}+\mathcal{O}(2 \sqrt{\pi}-v), \quad v \uparrow 2 \sqrt{\pi} .
$$

When we use Darboux's method in a first order form, we get the leading asymptotics of $a_{n}$ as

$$
a_{n}=\frac{\sin \frac{\pi}{4}(n-1)}{\left(\frac{1}{2} n\right)^{3 / 2}(2 \sqrt{\pi})^{n}}+\text { lower order. }
$$


Fig. 3 The ratio of $a_{n}$ and the approximation in (154), minus 1 , for the first 40 coefficients

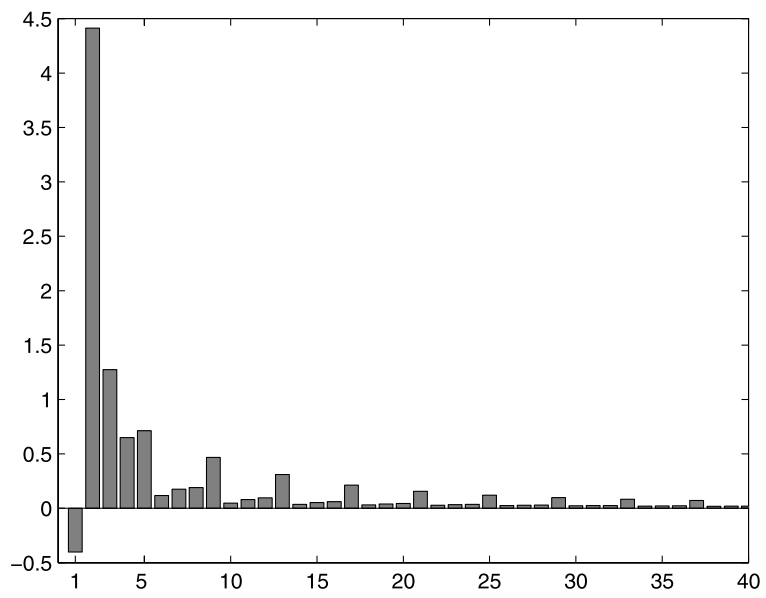

Note that the leading term at the right-hand side of (158) vanishes when $n=4 k+1$, $k=0,1, \ldots$, and so more precise information is required. This can be obtained when we write

$$
-y\left(e^{\pi i / 4} v\right)-\ln \left(1-y\left(e^{\pi i / 4} v\right)\right)=\frac{1}{2}\left(e^{-\pi i / 4}\left(4 \pi-v^{2}\right)^{1 / 2}\right)^{2},
$$

so that from $y(x)=\sum_{n=1}^{\infty} a_{n} x^{n}$ we get

$$
y\left(e^{\pi i / 4} v\right)=\sum_{n=1}^{\infty} a_{n}\left(e^{-\pi i / 4}\left(4 \pi-v^{2}\right)^{1 / 2}\right)^{n}
$$

and a similar expression for $y\left(e^{-\pi i / 4} v\right)$. We are now in a position to use the full strength of Darboux's theorem, see Theorem 8.4 in [29] (noting that it should be $-e^{-i \varphi_{k}}$ instead of $-e^{i \varphi_{k}}$ at the right-hand side of (8.4.8)). For instance, from the fact that $a_{2}=-1 / 3$, we get (154). The particular form $2 \pi /(6 n-9)$ of the coefficient in front of $\cos \frac{\pi}{4}(n-1)$ arises when the binomials occurring in Darboux's results are manipulated; also it has been used that $(-1)^{n}\left(\begin{array}{c}1 / 2 \\ n\end{array}\right)$ is well approximated by $-1 /((2 n-1) \sqrt{\pi(n+1 / 2)})$. Note that the leading factor in (154) is non-vanishing for any $n=1,2, \ldots$.

The accuracy of the approximation (154) is demonstrated by Fig. 3.

\section{Lemma 14 It holds that}

$$
y(x)=1-\sum_{m=1}^{\infty} \frac{m^{m-1}}{m ! e^{m}} e^{-\frac{1}{2} m x^{2}}, \quad|\arg (x)| \leq \pi / 4 .
$$


Proof Letting $z=1-y$ and $u=\exp \left(-1-\frac{1}{2} x^{2}\right)$, we can write $-y-\ln (1-y)=\frac{1}{2} x^{2}$ as

$$
z e^{-z}=u,
$$

so that by the Bürmann-Lagrange inversion formula

$$
z(u)=\left.\sum_{m=1}^{\infty} \frac{1}{m !}\left(\frac{\mathrm{d}}{\mathrm{d} z}\right)^{m-1}\left(\frac{z}{z e^{-z}}\right)^{m}\right|_{z=0} u^{m}=\sum_{m=1}^{\infty} \frac{m^{m-1}}{m !} u^{m} .
$$

The right-hand side of (163) is analytic in $|u|<1 / e$ and continuous in $|u| \leq 1 / e$. This leads to the formula in (161), with $x$ restricted to $|\arg (x)| \leq \pi / 4$.

An interesting consequence of (161) is that

$$
\begin{aligned}
\int_{a}^{\infty} e^{-\frac{1}{2} l s x^{2}} y^{\prime}(x) \mathrm{d} x & =\int_{a}^{\infty} e^{-\frac{1}{2} l s x^{2}} \sum_{m=1}^{\infty} \frac{m^{m-1}}{m ! e^{m}} \cdot m x \cdot e^{-\frac{1}{2} m x^{2}} \mathrm{~d} x \\
& =\sum_{m=1}^{\infty} \frac{m^{m}}{m ! e^{m}} \int_{a}^{\infty} e^{-\frac{1}{2}(m+l s) x^{2}} x \mathrm{~d} x \\
& =\sum_{m=1}^{\infty} \frac{m^{m} e^{-\frac{1}{2}(m+l s) a^{2}}}{m ! e^{m}(m+l s)} .
\end{aligned}
$$

This series expansion can be then be inserted into (55) to yield

$$
G_{k}(a)=\sum_{m=1}^{\infty} \frac{m^{m}}{m !} e^{-\frac{1}{2} m\left(a^{2}+2\right)} \sum_{l=1}^{\infty} \frac{l^{k+1 / 2}}{m+l s} e^{-\frac{1}{2} l s a^{2}} .
$$

For somewhat larger values of $a$, this seems to offer an attractive alternative to evaluate the $G_{k}(a)$ numerically. Furthermore, (161) can be used to establish a formal connection between the function $y$ and Lerch's transcendent. Indeed, we have

$$
\frac{k^{k-1}}{k ! e^{k}}=\frac{p(k)}{k \sqrt{2 \pi k}} \sim \frac{1}{\sqrt{2 \pi}}\left(\frac{1}{k^{3 / 2}}-\frac{1}{12 k^{5 / 2}}+\frac{1}{288 k^{7 / 2}}+\cdots\right) .
$$

Inserting this asymptotic expansion into (161) and using the definition (61) of Lerch's transcendent, we find a formal identity between $1-y(x)$ and

$$
\frac{e^{-\frac{1}{2} x^{2}}}{\sqrt{2 \pi}}\left(\Phi\left(e^{-\frac{1}{2} x^{2}}, \frac{3}{2}, 1\right)-\frac{1}{12} \Phi\left(e^{-\frac{1}{2} x^{2}}, \frac{5}{2}, 1\right)+\frac{1}{288} \Phi\left(e^{-\frac{1}{2} x^{2}}, \frac{7}{2}, 1\right)+\cdots\right) .
$$

Observe that each of the $\Phi$-functions in (167) has a convergent power series in $x$, $|x|<2 \sqrt{\pi}$, per formula (63).

We conclude our analysis of $y$ with the following result. 
Lemma 15 There is the inequality

$$
1-\frac{2}{3} x \leq y^{\prime}(x) \leq 1, \quad x \geq 0
$$

Proof Solve $f(y)=-y-\ln (1-y)=\frac{1}{2} x^{2}$ for the unique solution $y(x) \in[0,1)$. From the inequalities, to be proved below,

$$
-\frac{x}{1+x / 3}-\ln \left(1-\frac{x}{1+x / 3}\right) \geq \frac{1}{2} x^{2} \geq-\frac{x}{1+x}-\ln \left(1-\frac{x}{1+x}\right), \quad x \geq 0,
$$

and monotonicity of $f$ we infer that

$$
\frac{x}{1+x / 3} \geq y(x) \geq \frac{x}{1+x}, \quad x \geq 0
$$

From (151) we get

$$
y^{\prime}(x)=x\left(\frac{1}{y(x)}-1\right), \quad x \geq 0 .
$$

Inserting the inequalities (170) into (171), we find

$$
1-\frac{2}{3} x=x\left(\frac{1+x / 3}{x}-1\right) \leq y^{\prime}(x) \leq x\left(\frac{1+x}{x}-1\right)=1, \quad x \geq 0,
$$

as required. We now prove the two inequalities in (169). As to the second one, we need to show that

$$
g_{2}(x):=-x+(1+x) \ln (1+x) \leq \frac{1}{2} x^{2}+\frac{1}{2} x^{3}=: h_{2}(x), \quad x \geq 0 .
$$

Noting that $g_{2}(0)=h_{2}(0)=0, g_{2}^{\prime}(0)=h_{2}^{\prime}(0)=0$, and

$$
g_{2}^{\prime \prime}(x)=\frac{1}{1+x} \leq 1+\frac{3}{2} x=h_{2}^{\prime \prime}(x), \quad x \geq 0,
$$

we get (173). Note that the second inequality in (169) is the sharpest in its kind: when $\alpha>0$, the inequality $y(x) \geq x /(1+(1-\alpha) x)$ fails to hold for large $x \geq 0$, since this would require

$$
-\frac{x}{1+(1-\alpha) x}-\ln \left(\frac{1-\alpha x}{1+(1-\alpha) x}\right) \leq \frac{1}{2} x^{2}, \quad 0 \leq x<\infty,
$$

to hold. We next show the first inequality in (169). To that end we must prove that

$$
\begin{aligned}
g_{1}(x) & :=-x-(1+x / 3)(\ln (1-2 x / 3)-\ln (1+x / 3)) \\
& \geq \frac{1}{2} x^{2}+\frac{1}{6} x^{3}=: h_{1}(x), \quad x \geq 0 .
\end{aligned}
$$


It holds that $g_{1}(0)=h_{1}(0)=0, g_{1}^{\prime}(0)=h_{1}^{\prime}(0)=0, g_{1}^{\prime \prime}(0)=h_{1}^{\prime \prime}(0)=1$, while

$$
g_{1}^{\prime \prime \prime}(x)=\frac{4 / 27}{(1-2 x / 3)^{2}}-\frac{1 / 27}{(1+x / 3)^{2}}+\frac{8 / 9}{(1-2 x / 3)^{3}} \geq 1=h_{3}^{\prime \prime \prime}(x), \quad x \geq 0,
$$

again with equality at $x=0$. This proves the lemma.

\section{References}

1. Abramowitz, M., Stegun, I.A.: Handbook of Mathematical Functions, 9th edn. Dover, New York (1970)

2. Asmussen, S.: Applied Probability and Queues, 2nd edn. Springer, New York (2003)

3. Barndorff-Nielsen, O.E., Cox, D.R.: Asymptotic Techniques for Use in Statistics. Chapman and Hall, London (1990)

4. Borst, S., Mandelbaum, A., Reiman, M.: Dimensioning large call centers. Oper. Res. 52, 17-34 (2004)

5. Blanchet, J., Glynn, P.: Complete corrected diffusion approximations for the maximum of a random walk. Ann. Appl. Probab. 16, 951-983 (2006)

6. Chang, J.T., Peres, Y.: Ladder heights, Gaussian random walks and the Riemann zeta function. Ann. Probab. 25, 787-802 (1997)

7. Corless, R.M., Gonnet, G.H., Hare, D.E.G., Jeffrey, D.J., Knuth, D.E.: On the Lambert W function. Adv. Comput. Math. 5, 329-359 (1996)

8. De Bruijn, N.G.: Asymptotic Methods in Analysis. Dover, New York (1981)

9. Erdélyi, A., Magnus, W., Oberhettinger, F., Tricomi, F.G.: Higher Transcendental Functions, vol. I. McGraw-Hill, New York (1953)

10. Franx, G.J.: A simple solution for the M/D/c waiting time distribution. Oper. Res. Lett. 29, 221-229 (2001)

11. Gans, N., Koole, G., Mandelbaum, A.: Telephone call centers: tutorial, review and research prospects. Manuf. Serv. Oper. Manag. 5, 79-141 (2003)

12. Halfin, S., Whitt, W.: Heavy-traffic limits for queues with many exponential servers. Oper. Res. 29, 567-588 (1981)

13. Janssen, A.J.E.M., van Leeuwaarden, J.S.H.: On Lerch's transcendent and the Gaussian random walk. Ann. Appl. Probab. 17, 421-439 (2007)

14. Janssen, A.J.E.M., van Leeuwaarden, J.S.H.: Cumulants of the maximum of the Gaussian random walk. Stoch. Process. Appl. 117, 1928-1959 (2007)

15. Janssen, A.J.E.M., van Leeuwaarden, J.S.H., Zwart, B.: Refining square root staffing by expanding Erlang C. Oper. Res. (2008, under revision)

16. Jeffrey, D.J., Hare, D.E.G., Corless, R.M.: Unwinding the branches of the Lambert W function. Math. Sci. 21, 1-7 (1996)

17. Jagerman, D.: Some properties of the Erlang loss function. Bell Syst. Tech. J. 53, 525-551 (1974)

18. Jelenković, P., Mandelbaum, A., Momčilovic, P.: Heavy traffic limits for queues with many deterministic servers. Queueing Syst. 47, 53-69 (2004)

19. Jensen, J.L.: Saddlepoint Approximations. Clarendon Press, Oxford (1995)

20. Mandelbaum, A., Momčilovic, P.: Queues with many servers: The virtual waiting-time process in the QED regime (2005)

21. Mandelbaum, A.: http://iew3.technion.ac.il/serveng/References/references.html

22. Michel, R.: On Berry-Esseen bounds for the compound Poisson distribution. Insur. Math. Econ. 13, 35-37 (1993)

23. Olver, F.W.J.: Asymptotics and Special Functions. Academic, New York (1974)

24. Puhalskii, A., Reiman, M.: The multiclass GI/PH/N queue in the Halfin-Whitt regime. Adv. Appl. Probab. 32, 564-595 (2000)

25. Reed, J.: The $G / G I / N$ queue in the Halfin-Whitt regime. Preprint (2006)

26. Siegmund, D.: Corrected diffusion approximations in certain random walk problems. Adv. Appl. Probab. 11, 701-719 (1979) 
27. Spitzer, F.L.: A combinatorial lemma and its application to probability theory. Trans. Am. Math. Soc. 82, 323-339 (1956)

28. Szegö, G.: Über eine Eigenschaft der Exponentialreihe. Sitzungsber. Berl. Math. Ges. 22, 50-64 (1922)

29. Szegö, G.: Orthogonal Polynomials, 4th edn. American Mathematical Society, Providence (1975)

30. Temme, N.M.: The uniform asymptotic expansion of a class of integrals related to cumulative distribution functions. SIAM J. Math. Anal. 13, 239-253 (1982)

31. Temme, N.M.: Special Functions. Wiley, New York (1996)

32. Tijms, H.C.: Stochastic Models: An Algorithmic Approach. Wiley, New York (1994)

33. van Leeuwaarden, J.S.H.: Queueing models for cable access networks. Ph.D. thesis, Eindhoven University of Technology, The Netherlands (2005)

34. Watson, G.N.: An expansion related to Stirling's formula, derived by the method of steepest descent. Q. J. Pure Appl. Math. 48, 1-18 (1920)

35. Whitt, W.: Heavy traffic limit theorems for the $G / H_{2}^{*} / n / m$ queue. Math. Oper. Res. 30, 1-27 (2005) 